Guthrie, D. G.P., Torabi, M. and Karimi, N. (2019) Energetic and entropic analyses of double-diffusive, forced convection heat and mass transfer in microreactors assisted with nanofluid. Journal of Thermal Analysis and Calorimetry, 137(2), pp. 637-658. (doi:10.1007/s10973-018-7959-3)

There may be differences between this version and the published version. You are advised to consult the publisher's version if you wish to cite from it.

http://eprints.gla.ac.uk/174347/

Deposited on: 29 November 2018

Enlighten - Research publications by members of the University of Glasgow http://eprints.gla.ac.uk 


\title{
Energetic and entropic analyses of double-diffusive, forced convection heat and mass transfer in microreactors assisted with nanofluid
}

\author{
David G.P. Guthrie a, Mohsen Torabi ${ }^{1, b}$, Nader Karimi 1,a,c \\ a School of Engineering, University of Glasgow, Glasgow G12 8QQ, United Kingdom \\ b School of Engineering, University of California, Merced, CA 95348, USA \\ c Civil and Mechanical Engineering Department, University of Missouri-Kansas City, \\ Kansas City, MO 64110, USA
}

\begin{abstract}
This paper investigates the energetic and entropic characteristics of a microchannel with thick walls. A first order, catalytic chemical reaction is imposed on the inner surfaces of the microchannel walls and local thermal non-equilibrium approach is employed to analyse heat transfer within the porous section of the microchannel. Further, endo/exothermic physicochemical processes are incorporated into the fluid phase and solid structure of the microchannel. Two models describing the fluid-porous interface conditions known as Models A and B are incorporated. It is shown that for both interface models, and with the considered parametric values, the optimum thickness of the porous insert to achieve the maximum $\mathrm{Nu}$ is around 0.6. However, when $\mathrm{PEC}$ is considered this optimum thickness may vary between 0 and 0.5 . It is further shown that depending on the specification of the microreactor, either of Models A or B may result in the prediction of the minimum total entropy generation rate. It is also demonstrated that by altering the endothermicity of the microreactor it is possible to find an optimal value, which minimises the total rate of entropy generation.
\end{abstract}

\footnotetext{
${ }^{1}$ Corresponding authors.

E-mails: Mohsen.Torabi@my.cityu.edu.hk, mtorabi@ucmrced.edu (M. Torabi), Nader.Karimi@glasgow.ac.uk (N.Karimi).
} 
Keywords: Microreactor; Catalytic reactions; Porous material; Entropy generation; Local thermal non-equilibrium; Performance evaluation criterion.

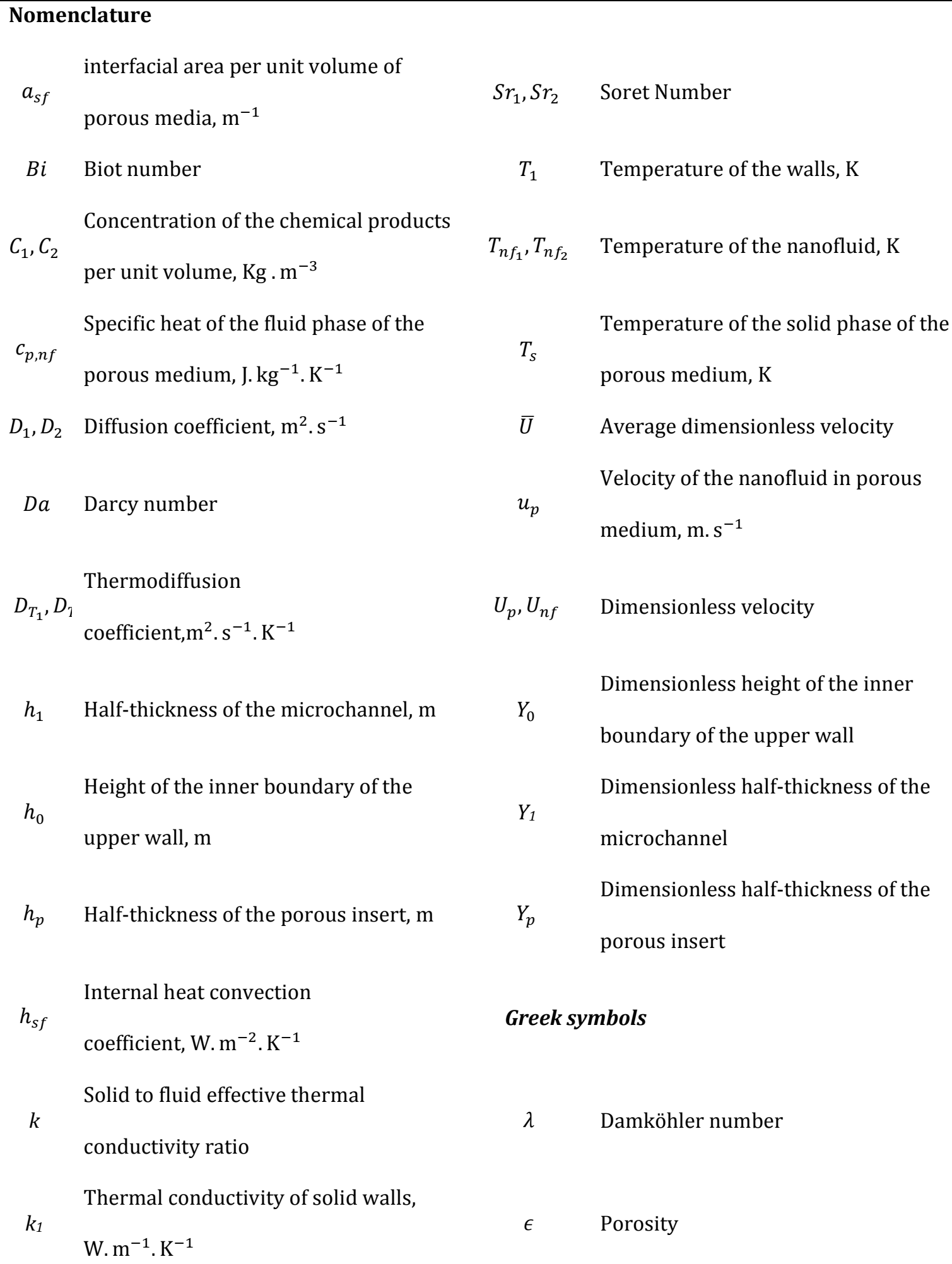

$S r_{1}, S r_{2} \quad$ Soret Number

$T_{1} \quad$ Temperature of the walls, $\mathrm{K}$

$T_{n f_{1}}, T_{n f_{2}} \quad$ Temperature of the nanofluid, $\mathrm{K}$

$T_{S}$ Temperature of the solid phase of the porous medium, $\mathrm{K}$

$\bar{U} \quad$ Average dimensionless velocity Velocity of the nanofluid in porous medium, m. $\mathrm{s}^{-1}$

$U_{p}, U_{n f} \quad$ Dimensionless velocity $Y_{0}$ Dimensionless height of the inner boundary of the upper wall

$Y_{1}$ Dimensionless half-thickness of the microchannel Dimensionless half-thickness of the porous insert

\section{Greek symbols}

$\lambda \quad$ Damköhler number

$\epsilon \quad$ Porosity 


\begin{tabular}{|c|c|c|c|}
\hline$k_{1 s}$ & $\begin{array}{l}\text { Ratio of the porous solid phase to solid } \\
\text { wall thermal conductivities }\end{array}$ & $\theta_{1}$ & $\begin{array}{l}\text { Dimensionless temperature of the solid } \\
\text { walls }\end{array}$ \\
\hline$k_{e, f}$ & $\begin{array}{l}\text { Effective thermal conductivity of the } \\
\text { fluid phase of the porous medium, } \\
\mathrm{W} \cdot \mathrm{m}^{-1} \cdot \mathrm{K}^{-1}\end{array}$ & $\theta_{n f}$ & $\begin{array}{l}\text { Dimensionless temperature of the } \\
\text { nanofluid phase }\end{array}$ \\
\hline$k_{e, s}$ & $\begin{array}{l}\text { Effective thermal conductivity of the } \\
\text { solid phase of the porous medium, } \\
\text { W. } \mathrm{m}^{-1} \cdot \mathrm{K}^{-1}\end{array}$ & $\theta_{m}$ & $\begin{array}{l}\text { Dimensionless average temperature of } \\
\text { the nanofluid phase }\end{array}$ \\
\hline$k_{f}$ & $\begin{array}{l}\text { Thermal conductivity of the base fluid, } \\
\mathrm{W} \cdot \mathrm{m}^{-1} \cdot \mathrm{K}^{-1}\end{array}$ & $\theta_{s}$ & $\begin{array}{l}\text { Dimensionless temperature of the solid } \\
\text { phase of the porous medium }\end{array}$ \\
\hline$k_{n f}$ & $\begin{array}{l}\text { Thermal conductivity of the nanofluid, } \\
\text { W. } \mathrm{m}^{-1} \cdot \mathrm{K}^{-1}\end{array}$ & $\kappa$ & Permeability, $\mathrm{m}^{2}$ \\
\hline$k_{p}$ & $\begin{array}{l}\text { Thermal conductivity of the } \\
\text { nanoparticles, } \mathrm{W} \cdot \mathrm{m}^{-1} \cdot \mathrm{K}^{-1}\end{array}$ & $\mu_{e, n f}$ & $\begin{array}{l}\text { Dynamic viscosity of porous medium, } \\
\mathrm{Kg} \cdot \mathrm{s}^{-1} \cdot \mathrm{m}^{-1}\end{array}$ \\
\hline$k_{d}$ & Reaction kinetic constant, $\mathrm{m} . \mathrm{s}^{-1}$ & $\mu_{f}$ & $\begin{array}{l}\text { Dynamic viscosity of the base fluid, } \\
\text { Kg. } \mathrm{s}^{-1} \cdot \mathrm{m}^{-1}\end{array}$ \\
\hline $\mathrm{Nu}$ & Nusselt Number & $\mu_{n f}$ & $\begin{array}{l}\text { Dynamic viscosity of the nanofluid, } \\
\text { Kg. } \mathrm{s}^{-1} \cdot \mathrm{m}^{-1}\end{array}$ \\
\hline$N_{s}$ & $\begin{array}{l}\text { Dimensionless local entropy } \\
\text { generation rate }\end{array}$ & $\omega_{s}$ & $\begin{array}{l}\text { Dimensionless volumetric internal heat } \\
\text { generation rate for the solid phase of the } \\
\text { porous medium }\end{array}$ \\
\hline$N_{t}$ & $\begin{array}{l}\text { Dimensionless total entropy } \\
\text { generation rate }\end{array}$ & $\omega_{n f}$ & $\begin{array}{l}\text { Dimensionless volumetric internal heat } \\
\text { generation rate for the nanofluid phase }\end{array}$ \\
\hline$p$ & Pressure, Pa & $\rho_{n f}$ & density of the nanofluid phase, $\mathrm{kg} \cdot \mathrm{m}^{-3}$ \\
\hline$Q_{1}$ & $\begin{array}{l}\text { Dimensionless volumetric internal heat } \\
\text { generation rate for the solid walls }\end{array}$ & $\Phi_{1} . \Phi_{2}$ & Dimensionless concentration \\
\hline$\dot{q}_{1}$ & $\begin{array}{l}\text { Volumetric internal heat generation } \\
\text { rate for the solid wall, } \mathrm{W} . \mathrm{m}^{-3}\end{array}$ & $\psi_{1} \cdot \psi_{2}$ & $\begin{array}{l}\text { Dimensionless parameters associated } \\
\text { with concentration used in entropy } \\
\text { generation equation }\end{array}$ \\
\hline
\end{tabular}




\begin{tabular}{|rlrl}
\hline \multicolumn{1}{|c|}{ Volumetric internal heat generation } & & Dimensionless parameters associated \\
$S_{S} \quad$ & rate for the solid phase of the porous & $\tau$ & with temperature used in entropy \\
& medium, $\mathrm{W} \cdot \mathrm{m}^{-3}$ & generation equation \\
$S_{n f}$ & Volumetric internal heat generation & \\
& rate for the nanofluid phase, $\mathrm{W} \cdot \mathrm{m}^{-3}$ & \\
$S^{\prime \prime \prime}$ & Local entropy generation rate, \\
& W. $\mathrm{K}^{-1} \cdot \mathrm{m}^{-3}$
\end{tabular}

\section{Introduction}

Besides the first law analyses of energy systems, the second law investigations of these systems have gained considerable attention. This is because the latter provides a quantitative insight into the system performance in addition to the qualitative information provided by the former [1]. The second law of thermodynamics uses both temperature distribution and heat flux specifications of the system [2][3], and hence is capable of providing more informative illustration of an energy system. Over the last two decades, a wide range of thermo-mechanical systems have been investigated from the second-law viewpoints [4][5]. However, extension of these analyses to thermochemical systems has been slow and only gained some momentum in recent years [6].

The heat transfer analyses of microchannels have been the focus of a large number of investigations during several past years, see for example [7][8][9][10]. Recently, the second law analyses of such systems have also received significant attention from the research community [11][6]. This is because microchannels are the building blocks of various small-scale thermofluid and thermochemical systems including microreactors, with numerous applications in energy and other technological fields. For example, microchannels find applications in heat sinks [12], single-phase [13] and multi-phase [14] heat transfer, electroosmotic and pressure driven flows [15][16], and fuel cells [17]. 
Microreactors consisting of a bundle of microchannels [18], have recently attracted considerable interests due to their various applications in chemical and pharmaceutical industries for process intensification [19][20], multiphase reactions [21] and, fast and exothermic/endothermic reactions [22][23][24][25]. Given these extensive application area, it is clear that the accurate predictions of energetic and entropic characteristics of microreactors are in growing demand.

So far, a handful of second law investigations on microchannels, with or without chemical reactions, have been performed. Although the pioneering studies considered negligible thickness for the walls of the microchannel [26][27][28], recently the conduction heat transfer via walls has been incorporated into the thermal analyses [29][30]. It has been shown that the incorporation of walls thickness within the energy equation is essential and has substantial effects on the predicted temperature distributions within microchannels [31][11]. These investigations have been not only on the pure heat transfer analyses [29], but also on double-diffusive heat and mass transfer visualizations [32]. It has been also shown that not only the pressure drop is reduced if the porous insert partially fills the microchannel, but also heat transfer in the microchannel can be maximized by using the optimal thickness of the porous insert [33][34]. While not directly the effect of porous thickness on pressure drop was considered on the performance of an annular pipe, it was comprehensively investigated against the performance number [35]. Also, the analyses have been extended to the effect of non-homogenous porous foams on the performance of channel-like thermal systems [36]. Ultimately speaking, this would reduce the total entropy generation of the system, for the heat transfer rate, as the pumping losses are reduced. Porous materials have been already incorporated into microchannels [37] and catalytic microreactors [38], and some second law investigations have been reported [6]. Nevertheless, the latter has, so far, 
remained limited to a few cases of fully filled microchannels [20][30][39]. It may be pointed out that, on the general field of heat and mass transfer, other investigations have been perform on the analysis of double-diffusive system such as Ref. [40]. Also, effects of absorber plate on performance of solar collector when the working fluid is the pure water has been considered in Ref. [41].

By comparing the predicted temperature fields through taking local thermal equilibrium (LTE) and local thermal non-equilibrium (LTNE) approaches, it has been shown that the resultant temperature distributions and Nusselt numbers can feature considerable discrepancies [6][42]. The differences between the findings of LTE and LTNE, i.e. the temperature difference between the fluid and solid phases of the porous section, have been particularly significant when internal heat generations are included [43][30]. Hence, more attention are being paid to the energetic and entropic investigations on porous microchannels while LTNE models have been practiced [44][39]. However, there is still a shortage of analyses on catalytic microreactors. Although a number of studies on the first and second law analyses of micro catalytic reactors have recently emerged [39][30][45], the influences of porous inserts within the microreactor has not been thoroughly investigated yet.

The current investigation aims to bridge the identified gaps in the literature on the transport and entropy generation in microreactor by considering thick walls and a centrally located porous insert in a catalytic microreactor. In an attempt to provide more accurate analyses of transport, LTNE is used to model heat transfer in the porous section, and Soret effect is included in the dispersion equations. Further, to enhance the heat transfer rate, nanoparticles are added to the base fluid [46].

\section{Theoretical methods}

\subsection{Problem configuration and assumptions}


A thick-walled microchannel, which represents the building block of thermochemical microreactors, is shown in Fig. 1. The microchannel includes two parallel plates and an open region through which fluid can flow freely. Also, a strip of porous material has been inserted along its centreline, which helps developing more uniform temperature and species distributions. In addition, nanoparticles may be added to the base fluid to boost the heat transfer process. Internal heat generations or consumption of a physical or a chemical nature has been assumed for all parts of the microchannel. The external surface of each wall is subject to a constant heat flux, and the internal surfaces of the walls are coated with a catalytic material, which provides a first order chemical reaction. The microchannel is axisymmetric about its centreline and therefore only half of the configuration is under analysis. In Fig. 1 , the parameter $h_{p}$ denotes the distance from the centreline to the edge of the porous insert, $h_{0}$ shows the distance to the inner surface of the wall, and $h_{1}$ represents the distance to the boundary of the configuration. The subsequent analyses rely on the following assumptions.

- The porous material is homogeneous and isotropic and the porous region is under local thermal non-equilibrium.

- The flow is incompressible, steady and laminar, and is thermally and hydrodynamically fully developed.

- Nanoparticles are distributed throughout the flow uniformly.

- Internal heat generation and/or consumption occurs uniformly in each component of the system according to the provided rates.

- All physical properties of the system (i.e. porosity, density, thermal conductivity, nanoparticle concentration and specific heat) are invariants. Also, the interstitial heat transfer coefficient within the porous phase of the system remains constant within the entire system [34][47]. 
- Radiation and natural convection are considered negligible.

- The transportation of species occurs through the mechanisms of Fickian and thermal diffusion of mass (Soret effect), while the mass transfer Peclet number is small.

\subsection{Governing equations}

Considering the stated assumptions together with Darcy-Brinkman flow model for the porous section of the microchannel the following equations govern the transport of momentum in the system.

$$
\begin{gathered}
-\frac{\partial p}{\partial x}+\mu_{n f} \frac{\partial^{2} u_{f}}{\partial y^{2}}=0 \text { for } h_{p}<y<h_{0}, \\
-\frac{\partial p}{\partial x}+\mu_{e} \frac{\partial^{2} u_{p}}{\partial y^{2}}-\frac{\mu_{n f}}{K} u_{p}=0 \text { for } 0<y<h_{p} .
\end{gathered}
$$

Further, the following set of equations describe the transport of thermal energy throughout each of the system's components. They correspond, respectively, to the wall, open region, and nanofluid and solid phases of the porous region:

$$
\begin{gathered}
k_{1} \frac{d^{2} T_{1}}{d y^{2}}+q_{1}=0 \text { for } h_{0}<y<h_{1}, \\
\rho_{n f} c_{n f, p} u_{n f} \frac{\partial T_{n f 1}}{\partial x}=k_{n f} \frac{\partial^{2} T_{n f 1}}{\partial y^{2}}+S_{n f} \text { for } h_{p}<y<h_{0}, \\
\rho_{n f} c_{n f, p} u_{p} \frac{\partial T_{n f 2}}{\partial x}=k_{e, n f} \frac{\partial^{2} T_{n f 2}}{\partial y^{2}}+a_{s f} h_{s f}\left(T_{s}-T_{n f 2}\right)+S_{n f} \text { for } 0<y \\
<h_{p}, \\
0=k_{e, s} \frac{\partial^{2} T_{s}}{\partial y^{2}}-a_{s f} h_{s f}\left(T_{s}-T_{n f 2}\right)+S_{s} \text { for } 0<y<h_{p} .
\end{gathered}
$$

Considering the Fickian diffusion of species and the contributions of the Soret effect, the dispersion equations for both sections of the microchannel are expressed by the following relations. 


$$
\begin{aligned}
& D_{1} \frac{\partial^{2} C_{1}}{\partial y^{2}}+D_{T_{1}} \frac{\partial^{2} T_{n f 1}}{\partial y^{2}}=0 \text { for } h_{p}<y<h_{0} \\
& D_{2} \frac{\partial^{2} C_{2}}{\partial y^{2}}+D_{T_{2}} \frac{\partial^{2} T_{n f 2}}{\partial y^{2}}=0 \text { for } 0<y<h_{p}
\end{aligned}
$$

Furthermore, in order to investigate the irreversibilities introduced in the system, the following relations for local entropy generation rates are formulated [30][39][32].

$$
\dot{S}^{\prime \prime \prime}=\left\{\begin{array}{cc}
\frac{k_{e, s}}{T_{s}^{2}}\left[\left(\frac{\partial T_{s}}{\partial x}\right)^{2}+\left(\frac{\partial T_{s}}{\partial y}\right)^{2}\right]+\frac{h_{s f} a_{s f}\left(T_{s}-T_{n f 2}\right)^{2}}{T_{s} T_{n f 2}} & \text { for } 0<y<h_{p} \\
\frac{k_{e, n f}}{T_{n f 2}^{2}}\left[\left(\frac{\partial T_{n f 2}}{\partial x}\right)^{2}+\left(\frac{\partial T_{n f 2}}{\partial y}\right)^{2}\right]+\frac{h_{s f} a_{s f}\left(T_{s}-T_{n f 2}\right)^{2}}{T_{s} T_{n f 2}} & \text { for } 0<y<h_{p} \\
+\frac{\mu_{n f}}{k T_{n f 2}} u_{p}^{2}+\frac{\mu_{e, n f}}{T_{n f 2}}\left(\frac{\partial u_{p}}{\partial y}\right)^{2}+\frac{R D_{2}}{C_{2}}\left(\frac{\partial C_{2}}{\partial y}\right)^{2}+\frac{R D_{2}}{T_{n f 2}}\left(\frac{\partial C_{2}}{\partial y}\right)\left(\frac{\partial T_{n f 2}}{\partial y}\right) & \text { for } h_{p}<y<h_{0} \\
\frac{k_{n f}}{T_{n f 1}^{2}}\left[\left(\frac{\partial T_{n f 1}}{\partial x}\right)^{2}+\left(\frac{\partial T_{n f 1}}{\partial y}\right)^{2}\right]+\frac{\mu_{n f}}{k T_{n f 1}} u_{n f}^{2} & \frac{R D_{1}}{C_{1}}\left(\frac{\partial C_{1}}{\partial y}\right)^{2}+\frac{R D_{1}}{T_{n f 1}}\left(\frac{\partial C_{1}}{\partial y}\right)^{2}\left(\frac{\partial T_{n f 1}}{\partial y}\right) \\
\frac{k_{1}}{T_{1}^{2}}\left[\left(\frac{\partial T_{1}}{\partial x}\right)^{2}+\left(\frac{\partial T_{1}}{\partial y}\right)^{2}\right] \text { for } h_{0}<y<h_{1}
\end{array}\right.
$$

\subsection{Boundary equations}

The boundary conditions applied to the momentum equations are as follows.

$$
\begin{gathered}
\frac{\partial u_{p}}{\partial y}=0 \text { at } y=0 \\
u_{n f}=u_{p}, \quad \mu_{n f} \frac{\partial u_{n f}}{\partial y}=\mu_{e, n f} \frac{\partial u_{p}}{\partial y} \text { at } y=h_{p}, \\
u_{n f}=0 \text { at } y=h_{0},
\end{gathered}
$$

and those for the energy equations are:

$$
\begin{gathered}
\frac{\partial T_{n f 2}}{\partial y}=\frac{\partial T_{s}}{\partial y}=0 \text { at } y=0 \\
T_{n f 1}=T_{n f 2} \text { at } y=h_{p}
\end{gathered}
$$




$$
\begin{gathered}
T_{1}=T_{n f 1}, \quad k_{n f} \frac{\partial T_{n f 1}}{\partial y}=k_{1} \frac{\partial T_{1}}{\partial y}=\left.q\right|_{y=h_{0}} \text { at } y=h_{0}, \\
k_{1} \frac{\partial T_{1}}{\partial y}=q_{w} \text { at } y=h_{1} .
\end{gathered}
$$

The dispersion equations take the following boundary conditions.

$$
\begin{gathered}
\frac{\partial C_{2}}{\partial y}=0 \text { at } y=0, \\
C_{1}=C_{2}, \quad D_{1} \frac{\partial C_{1}}{\partial y}=D_{2} \frac{\partial C_{2}}{\partial y} \text { at } y=h_{p}, \\
D_{1} \frac{\partial C_{1}}{\partial y}=k_{d} C_{1} \text { at } y=h_{0} .
\end{gathered}
$$

Equation (12c) imposed the first order chemical reaction on the inner wall boundaries of the microreactor. On the fluid-porous interfaces, it is assumed that transfer of both species values and flux are constant, which lead to Eq. (12b).

There are two well-established models that describe the heat flux split over a porousfluid interface: Models 1A and 2A of Alazmi and Vafai [48], or Models A and B of Yang and Vafai [49]. Under Model A assumption, the total heat flux at the interface of the porous insert is the sum of the heat flux of each individual phase; defined by their effective thermal conductivities and temperature gradient, respectively:

$$
\begin{gathered}
q_{i n t}=\left.k_{e, n f} \frac{\partial T_{n f 2}}{\partial y}\right|_{y=h_{p}}+\left.k_{e, s} \frac{\partial T_{s}}{\partial y}\right|_{y=h_{p}}=\left.k_{n f} \frac{\partial T_{n f 1}}{\partial y}\right|_{y=h_{p}} \text { at } y=h_{p} . \\
T_{n f 1}=T_{n f 2}=T_{s} \text { at } y=h_{p}
\end{gathered}
$$

Alternatively, Model B assumes that both solid and fluid phases receive equal heat flux at the interface [48][49]:

$$
q_{i n t}=\left.k_{e, n f} \frac{\partial T_{n f 2}}{\partial y}\right|_{y=h_{p}}=\left.k_{e, s} \frac{\partial T_{s}}{\partial y}\right|_{y=h_{p}}=\left.k_{n f} \frac{\partial T_{n f 1}}{\partial y}\right|_{y=h_{p}} \text { at } y=h_{p} \text {. }
$$

Taking the average fluid velocity for the entire microchannel, defined as 


$$
\bar{u}=\frac{1}{h_{0}}\left[\int_{0}^{h_{p}} u_{p} d y+\int_{h_{p}}^{h_{0}} u_{f} d y\right],
$$

and recalling that for a fully developed flow, incorporating the assumptions stated in Section 2.1, $\frac{\partial T_{n f 1}}{\partial x}=\frac{\partial T_{n f 2}}{\partial x}=\frac{\partial T_{S}}{\partial x}=\frac{\partial T_{1}}{\partial x}=\frac{\partial T}{\partial x}$, derivations for the heat flux at the porous-fluid interface for either of the two interface models can be carried out. Firstly, by integrating the energy equation of each system component, provided by Eqs. (3)-(6), the following relation for the interfacial heat flux under Model A is derived.

$$
\begin{aligned}
\left.\frac{\left.q\right|_{y=h_{p}}}{q_{w}}\right|_{\text {Model } A} & =\frac{1}{h_{o} \bar{u}} \int_{0}^{h_{p}} u_{p} d y \\
& +\frac{1}{q_{w}}\left[\frac { 1 } { h _ { o } \overline { u } } \left(q_{1}\left(h_{1}-h_{0}\right) \int_{0}^{h_{p}} u_{p} d y+\left(S_{n f} h_{0}\right.\right.\right. \\
& \left.\left.\left.+S_{S} h_{p}\right) \int_{0}^{h_{p}} u_{p} d y\right)-h_{p}\left(S_{s}+S_{n f}\right)\right] .
\end{aligned}
$$

Similarly, applying Model B condition reveals

$$
\begin{gathered}
\left.\frac{\left.q\right|_{y=h_{p}}}{q_{w}}\right|_{\text {Model } B}=\frac{\int_{0}^{h_{p}} u_{p} d y}{2 h_{0} \bar{u}-\int_{0}^{h_{p}} u_{p} d y}+\frac{1}{2 h_{0} \bar{u}-\int_{0}^{h_{p}} u_{p} d y}\left[\frac { 1 } { q _ { w } } \left(h_{p}\left(S_{S}+S_{n f}\right)-\right.\right. \\
\left.\left.\frac{\int_{0}^{h_{p}} u_{p} d y}{h_{o} \bar{u}}\left(q_{1}\left(h_{1}-h_{0}\right)+S_{n f} h_{0}+S_{s} h_{p}\right)\right)\right] .
\end{gathered}
$$

Lastly, by rearranging the terms in Eq. (16), an expression for the heat flux at the fluidwall interface, valid for both Models A and B, is developed. This reads,

$$
\frac{\left.q\right|_{y=h_{0}}}{q_{w}}=1+\frac{q_{1}\left(h_{1}-h_{0}\right)}{q_{w}} .
$$

\section{Dimensionless parameters and non-dimensionalised equations}

To facilitate analytical progression, the following dimensionless parameters are introduced. 


$$
\begin{aligned}
& \left.\left.\theta\right|_{\text {Model A }} \quad \theta\right|_{\text {Model B }} \\
& =\frac{k_{e, s}\left(T-T_{\text {int }}\right)}{q_{w} h_{1}}, \quad=\frac{k_{e, s}\left(T-T_{s, i n t}\right)}{q_{w} h_{1}}, \quad \gamma_{p}=\frac{\left.q\right|_{y=h_{p}}}{q_{w}}, \\
& \gamma_{s}=\frac{\left.q\right|_{y=h_{0}}}{q_{w}}, \quad k=\frac{k_{e, s}}{k_{e, f}}, \quad k_{1 s}=\frac{k_{1}}{k_{e, s}}, \\
& B i=\frac{a_{s f} h_{s f} h_{0}^{2}}{k_{e, s}}, \quad Y=\frac{y}{h_{1}}, \quad Y_{p}=\frac{h_{p}}{h_{1}} \\
& Y_{0}=\frac{h_{0}}{h_{1}}, \quad U=\frac{u}{u_{r}}, \quad Q_{1}=\frac{q_{1} h_{1}}{q_{w}}, \\
& \omega_{n f}=\frac{S_{n f} h_{1}}{q_{w}}, \quad \omega_{s}=\frac{S_{s} h_{1}}{q_{w}}, \quad D a=\frac{\kappa}{h_{1}^{2}}, \\
& D_{21}=\frac{D_{2}}{D_{1}}, \quad \Phi_{1}=\frac{C_{1}}{C_{0}}, \quad \Phi_{2}=\frac{C_{2}}{C_{0}}, \\
& \lambda=\frac{k_{d} h_{1}}{D_{1}}, \quad S r_{1}=\frac{D_{T_{1}} q_{w} h_{1}}{D_{1} C_{0} k_{e, s}}, \quad S r_{2}=\frac{D_{T_{2}} q_{w} h_{1}}{D_{2} C_{0} k_{e, s}}, \\
& C_{\mu}=\frac{\mu_{n f}}{\mu_{f}}=\frac{1}{1-\phi^{2.5}}, \quad C_{k}=\frac{k_{n f}}{k_{f}}=1+\frac{3 \phi\left(\frac{k_{p}}{k_{f}}-1\right)}{\left(\frac{k_{p}}{k_{f}}+2\right)-\phi\left(\frac{k_{p}}{k_{f}}-1\right)}, \\
& B r=\frac{\mu_{n f} u_{r}^{2}}{q_{w} h_{1}}, \quad \quad N_{s}=\frac{\dot{S}^{\prime \prime \prime} h_{1}^{2}}{k_{e, s}}, \quad \quad P e=\frac{\rho_{n f} c_{n f, p} h_{1} u_{r}}{k_{e, f}}, \\
& \psi_{1}=\frac{R D_{1} C_{0}}{k_{e, s}}, \quad \quad \psi_{2}=\frac{R D_{2} C_{0}}{k_{e, s}},\left.\quad \tau\right|_{\text {Model } A}=\frac{T_{\text {int }} k_{e, s}}{q_{w} h_{1}}, \\
& \left.\tau\right|_{\text {Model B }}=\frac{T_{s, \text { int }} k_{e, s}}{q_{w} h_{1}} .
\end{aligned}
$$

Darcy number $(D a)$ describes the permeability of the porous medium relative to the size of the microchannel, while Biot number $(B i)$ represents the strength of the internal heat exchange between the nanofluid and solid phases in the porous region. As $B i$ increases, LTNE temperature predictions should approach those of LTE [42]. Soret number $(\mathrm{Sr})$ describes the relationship between the temperature gradient of the system 
and the concentration flux of the chemical species in the flow. Further, the Damköhler number $(\lambda)$ relates the chemical reaction rate to the rate of mass transfer in the system. The fluid property ratios $C_{k}$ and $C_{\mu}$ indicate the relationship between the base fluid and equivalent nanofluid, in terms of thermal conductivity and viscosity, respectively. The detailed description of thermophysical properties of the used nanofluid can be found in Ref. [43]. Moreover, the characteristic velocity of the flow $u_{r}$ is defined as $u_{r}=-\frac{h_{0}^{2}}{\mu_{e}} \frac{\partial P}{\partial x}$. By normalising the momentum equations, the following dimensionless relations are obtained:

$$
\begin{gathered}
0=1+C_{\mu} \frac{\partial^{2} U_{n f}}{\partial Y^{2}} \text { for } Y_{p}<Y<Y_{0}, \\
0=1+\frac{\partial^{2} U_{p}}{\partial Y^{2}}-\frac{C_{\mu}}{Y_{0} D a} U_{p} \text { for } 0<Y<Y_{p},
\end{gathered}
$$

with the following associated boundary conditions:

$$
\begin{gathered}
\frac{\partial U_{p}}{\partial Y}=0 \text { at } Y=0, \\
U_{n f}=U_{p}, \quad C_{\mu} \frac{\partial U_{n f}}{\partial Y}=\frac{\partial U_{p}}{\partial Y} \text { at } Y=Y_{p}, \\
U_{n f}=0 \text { at } Y=Y_{0} .
\end{gathered}
$$

The dimensionless average velocity of the fluid in the microchannel is given by:

$$
\bar{U}=\frac{D a Y_{0} Y_{p}}{C_{\mu}}+\frac{c}{Z} \sinh \left(Z Y_{p}\right)-\frac{1}{6 C_{\mu}}\left(Y_{0}^{3}-Y_{p}^{3}\right)+\frac{1}{2} b\left(Y_{0}^{2}-Y_{p}^{2}\right)+a\left(Y_{0}-Y_{p}\right)
$$

where $Z=\sqrt{C_{\mu} /\left(D a Y_{0}\right)}$ and constants $a, b$ and $c$ are provided by:

$a$

$$
\begin{gathered}
=-\frac{\left(-C_{\mu} Y_{0}^{2}+2 C_{\mu} Y_{0} Y_{p}\right) \cosh \left(Z Y_{p}\right)+\left(Z Y_{0}^{2} Y_{p}-2 D a Z Y_{0}^{2}-Z Y_{0} Y_{p}^{2}\right) \sinh \left(Z Y_{p}\right)}{2 C_{\mu}\left(C_{\mu} \cosh \left(Z Y_{p}\right)+\left(Z Y_{0}-Z Y_{p}\right) \sinh \left(Z Y_{p}\right)\right)} \\
b=-\frac{-2 C_{\mu} Y_{p} \cosh \left(Z Y_{p}\right)+\left(2 D a Z Y_{0}-Z Y_{0}^{2}+Z Y_{p}^{2}\right) \sinh \left(Z Y_{p}\right)}{\left.2 C_{\mu}\left(C_{\mu} \cosh \left(Z Y_{p}\right)+\left(Z Y_{0}-Z Y_{p}\right) \sinh \left(Z Y_{p}\right)\right]\right)}
\end{gathered}
$$




$$
c=-\frac{\left(2 D a Y_{0}-Y_{0}^{2}+2 Y_{0} Y_{p}-Y_{p}^{2}\right) \operatorname{csch}\left(Z Y_{p}\right)}{2\left(Z Y_{0}-Z Y_{p}+C_{\mu} \operatorname{coth}\left(Z Y_{p}\right)\right)} .
$$

For Model A, the normalised forms of the energy equations are given as follows,

$$
\begin{gathered}
\theta_{1}^{\prime \prime}(Y)+\frac{Q_{1}}{k_{1 s}}=0 \\
\frac{U_{n f}(Y)}{\bar{U}}\left(1+Q_{1}\left(1-Y_{0}\right)+\omega_{n f} Y_{0}+\omega_{s} Y_{p}\right)=\frac{C_{k} Y_{0}}{k \varepsilon} \theta_{n f 1}^{\prime \prime}(Y)+\omega_{n f} Y_{0} \\
\frac{U_{p}(Y)}{\bar{U}}\left(1+Q_{1}\left(1-Y_{0}\right)+\omega_{n f} Y_{0}+\omega_{s} Y_{p}\right) \\
=\frac{C_{k} Y_{0}}{k} \theta_{n f 2}^{\prime \prime}(Y)+\frac{B i}{Y_{0}}\left(\theta_{s}(Y)-\theta_{n f 2}(Y)\right)+\omega_{n f} Y_{0} \\
0=\theta_{s}^{\prime \prime}(Y)-\frac{B i}{Y_{0}}\left(\theta_{s}(Y)-\theta_{n f 2}(Y)\right)+\omega_{s}
\end{gathered}
$$

The above equations are bounded by the following conditions:

$$
\begin{gathered}
\theta_{n f 2}^{\prime}(0)=\theta_{s}^{\prime}(0)=0, \\
\theta_{n f 1}\left(Y_{p}\right)=\theta_{n f 2}\left(Y_{p}\right)=\theta_{s}\left(Y_{p}\right)=0, \\
\theta_{n f 1}\left(Y_{0}\right)=\theta_{1}\left(Y_{0}\right) \frac{C_{k}}{k \varepsilon} \\
\theta_{n f 1}^{\prime}\left(Y_{0}\right)=\theta_{1}^{\prime}\left(Y_{0}\right), \\
k_{1 s} \theta_{1}^{\prime}(1)=\frac{1}{k_{1 s}} .
\end{gathered}
$$

Similarly, under Model B the following energy equations are obtained,

$$
\begin{gathered}
\theta_{1}^{\prime \prime}(Y)+\frac{Q_{1}}{k_{1 s}}=0, \\
\frac{U_{n f}(Y)}{\bar{U}}\left(1+\left.\gamma_{p}\right|_{\text {Model } B}+Q_{1}\left(1-Y_{0}\right)+\omega_{n f} Y_{0}+\omega_{s} Y_{p}\right) \\
=\frac{C_{k} Y_{0}}{k \varepsilon} \theta_{n f 1}^{\prime \prime}(Y)+\omega_{n f} Y_{0},
\end{gathered}
$$




$$
\begin{gathered}
\frac{U_{p}(Y)}{\bar{U}}\left(1+\left.\gamma_{p}\right|_{\text {ModelB }}+Q_{1}\left(1-Y_{0}\right)+\omega_{n f} Y_{0}+\omega_{s} Y_{p}\right) \\
=\frac{C_{k} Y_{0}}{k} \theta_{n f 2}^{\prime \prime}(Y)+\frac{B i}{Y_{0}}\left(\theta_{s}(Y)-\theta_{n f 2}(Y)\right)+\omega_{n f} Y_{0} \\
0=\theta_{s}^{\prime \prime}(Y)-\frac{B i}{Y_{0}}\left(\theta_{s}(Y)-\theta_{n f 2}(Y)\right)+\omega_{s}
\end{gathered}
$$

with the following boundary conditions:

$$
\begin{gathered}
\theta_{n f 2}^{\prime}(0)=\theta_{s}^{\prime}(0)=0, \\
\theta_{n f 1}\left(Y_{p}\right)=\theta_{n f 2}\left(Y_{p}\right), \\
\theta_{s}\left(Y_{p}\right)=0, \\
\frac{C_{k}}{k} \theta_{n f 2}^{\prime}\left(Y_{p}\right)=\theta_{s}^{\prime}\left(Y_{p}\right)=\left.\gamma_{p}\right|_{\text {Model } B}, \\
\theta_{s}^{\prime \prime}\left(Y_{p}\right)+\frac{B i}{Y_{0}} \theta_{n f 2}\left(Y_{p}\right)+\omega_{s}=0, \\
\theta_{n f 1}\left(Y_{0}\right)=\theta_{1}\left(Y_{0}\right), \\
\frac{C_{k}}{k \varepsilon} \theta_{n f 1}^{\prime}\left(Y_{0}\right)=\theta_{1}^{\prime}\left(Y_{0}\right) k_{1 s}, \\
\theta_{1}^{\prime}(1)=\frac{1}{k_{1 s}} .
\end{gathered}
$$

The dimensionless forms of the dispersion equations are given by:

$$
\begin{aligned}
& \Phi_{2}^{\prime \prime}+S r_{2} \theta_{n f 2}^{\prime \prime}=0 \text { for } 0<Y<Y_{p}, \\
& \Phi_{1}^{\prime \prime}+S r_{1} \theta_{n f 1}^{\prime \prime}=0 \text { for } Y_{p}<Y<Y_{0},
\end{aligned}
$$

with the following boundary conditions:

$$
\begin{gathered}
\Phi_{2}^{\prime}=0 \text { at } Y=0, \\
\Phi_{1}=\Phi_{2}, \quad \Phi_{1}^{\prime}=D_{21} \Phi_{2}^{\prime} \text { at } Y=Y_{p}, \\
\Phi_{1}^{\prime}=\lambda \Phi_{1} \text { at } Y=Y_{0} .
\end{gathered}
$$

Furthermore, a prediction of $N u$ at the inside of the microchannel wall can be derived into the following form. 


$$
\begin{aligned}
& N u=\frac{4 \varepsilon k \gamma_{s}}{\theta_{w}-\theta_{m}} \text { for } Y_{p}<Y_{0}, \\
& N u=\frac{4 \varepsilon \gamma_{s}}{\theta_{w}-\theta_{m}} \text { for } Y_{p}=Y_{0},
\end{aligned}
$$

where $\theta_{w}=\left.\theta_{n f 1}\right|_{Y=Y_{0}}$ and $\theta_{m}$ represents the mean temperature in the microchannel,

defined as

$$
\theta_{m}=\frac{\int_{0}^{Y_{p}} U_{p} \theta_{n f 2} d y+\int_{Y_{p}}^{Y_{0}} U_{n f} \theta_{n f 1} d y}{\bar{U}}
$$

Substituting the variables defined in Eq. (19) into the entropy generation rate, the dimensionless local entropy generation rates are given by the following relation.

$N_{s}$

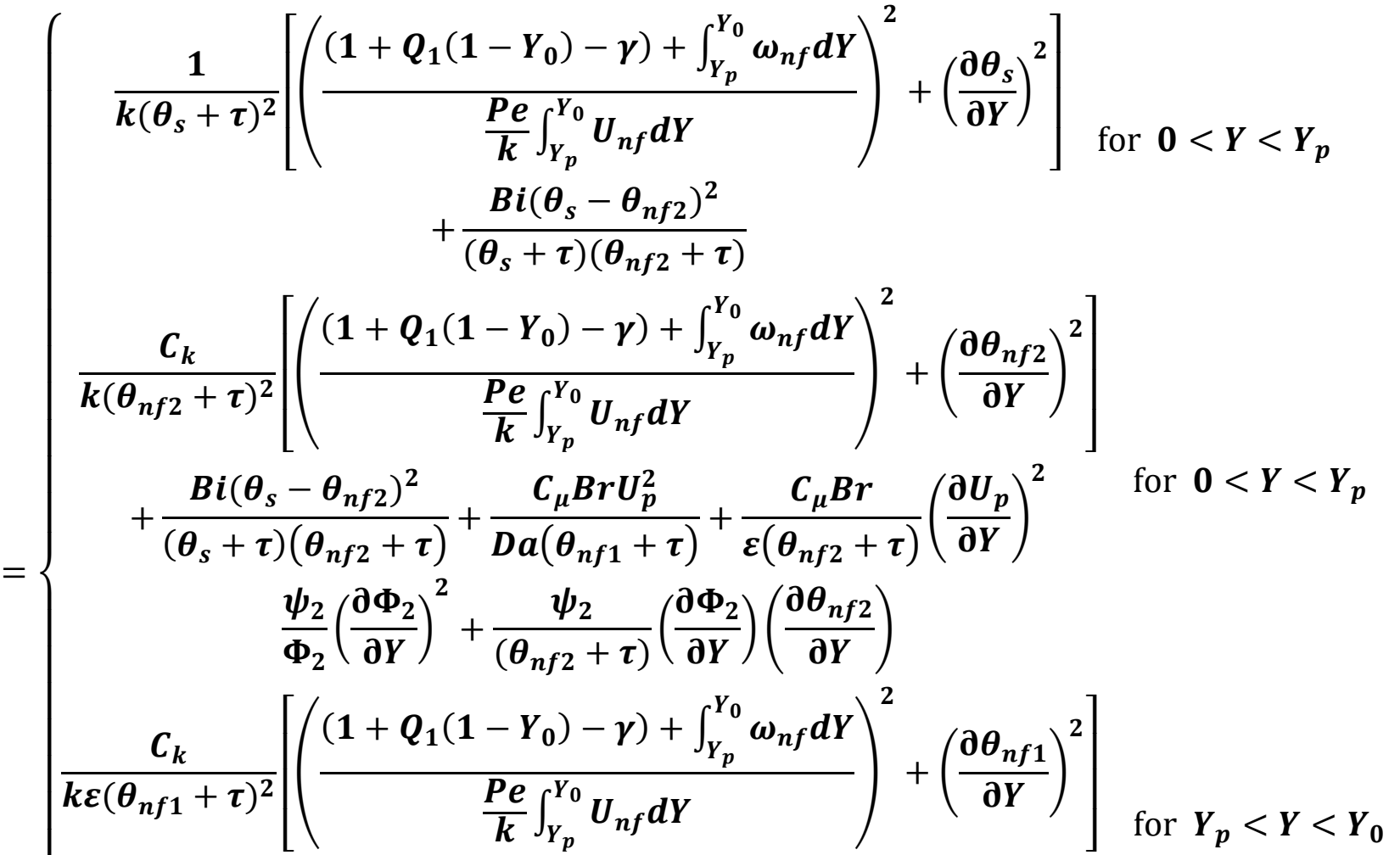

$$
\begin{aligned}
& +\frac{B r U_{n f 1}^{2}}{D a\left(\theta_{n f}+\tau\right)}+\frac{\psi_{1}}{\Phi_{1}}\left(\frac{\partial \Phi_{1}}{\partial Y}\right)^{2}+\frac{\psi_{1}}{\left(\theta_{n f 1}+\tau\right)}\left(\frac{\partial \Phi_{1}}{\partial Y}\right)\left(\frac{\partial \theta_{n f 1}}{\partial Y}\right) \\
& \frac{k_{1 s}}{\left(\theta_{1}+\tau\right)^{2}}\left[\left(\frac{\left(1+Q_{1}\left(1-Y_{0}\right)-\gamma\right)+\int_{Y_{p}}^{Y_{0}} \omega_{n f} d Y}{\frac{P e}{k} \int_{Y_{p}}^{Y_{0}} U_{n f} d Y}\right)^{2}+\left(\frac{\partial \theta_{1}}{\partial Y}\right)^{2}\right] \text { for } Y_{0}<Y<Y_{1}
\end{aligned}
$$


The total entropy generation rate is calculated by integrating the local entropy generation rate over the thickness of the half-microchannel,

$$
N_{t}=\int_{Y=0}^{Y=1} N_{S} d Y .
$$

Also, PEC provides an insight into both heat transfer and entropy generation performances of the microreactor and is calculated by [50]

$$
P E C=\frac{N u}{N_{t}}
$$

\section{Solution of momentum, energy and dispersion equations}

Through solution of the normalised transport equations presented in Section 3, the following expressions for the velocity, temperature and concentration fields can be obtained. Firstly, the velocity in the open and porous regions are given by:

$$
\begin{gathered}
U_{n f}(Y)=-\frac{Y^{2}}{2 C_{\mu}}+a+b Y, \\
U_{p}(Y)=\frac{D a Y_{0}}{C_{\mu}}+c \cosh (Z Y),
\end{gathered}
$$

where $Z=\sqrt{C_{\mu} /\left(D a Y_{0}\right)}$ and constants $a, b$ and $c$ are provided by Eq. (24). The energy equations were solved analytically by decoupling the equations pertaining to the porous region and subsequently applying additional boundary conditions derived from their second and third derivatives. The solution procedure is well-established and can be easily obtained through the literature [43][42]. This results in the following formulae for the wall, open region, and fluid and solid phases of the porous region, respectively.

Model A:

$$
\theta_{1}(Y)=-\frac{Q_{1} Y^{2}}{2 k_{1 s}}+A_{1}+A_{2} Y
$$




$$
\begin{gathered}
\theta_{n f 1}(Y)=Y^{3} b \frac{k \varepsilon \chi_{A}}{6 C_{k} \bar{U} Y_{0}}-Y^{4} \frac{k \varepsilon \chi_{A}}{24 C_{k} C_{\mu} \bar{U} Y_{0}}+Y^{2}\left(-\frac{k \omega_{n f} \varepsilon}{2 C_{k}}+a \frac{k \varepsilon \chi_{A}}{2 C_{k} \bar{U} Y_{0}}\right)+A_{3} \\
+A_{4} Y \\
\theta_{n f 2}(Y)=-\frac{k Y^{2}\left(C_{\mu} \bar{U} \omega_{s}+C_{\mu} \bar{U} \omega_{n f} Y_{0}-D a Y_{0} \chi_{A}\right)}{2 C_{\mu} \bar{U}\left(k+C_{k} Y_{0}\right)} \\
+A_{5} \frac{C_{k} Y_{0}^{2}}{B i\left(k+C_{k} Y_{0}\right)} \cosh (\alpha Y)+A_{6} \\
+c \frac{k\left(-B i+Y_{0} Z^{2}\right) \chi_{A}}{\bar{U} Z^{2}\left(-k B i-B i C_{k} Y_{0}+C_{k} Y_{0}^{2} Z^{2}\right)} \cosh (Z Y) \\
\theta_{s}(Y)=-\frac{k Y^{2}\left(C_{\mu} \bar{U} \omega_{s}+C_{\mu} \bar{U} \omega_{n f} Y_{0}-D a Y_{0} \chi_{A}\right)}{2 C_{\mu} \bar{U}\left(k+C_{k} Y_{0}\right)} \\
+A_{7} \frac{C_{k} Y_{0}^{2}}{B i\left(k+C_{k} Y_{0}\right)} \cosh (\alpha Y)+A_{8} \\
+c \frac{k B i \chi_{A}}{\bar{U} Z^{2}\left(k B i+B i C_{k} Y_{0}-C_{k} Y_{0}^{2} Z^{2}\right)} \cosh (Z Y)
\end{gathered}
$$

Model B:

$$
\begin{gathered}
\theta_{1}(Y)=-\frac{Q_{1} Y^{2}}{2 k_{1 s}}+B_{1}+B_{2} Y \\
\theta_{n f 1}(Y)=Y^{3} b \frac{k \varepsilon \chi_{B}}{6 C_{k} \bar{U} Y_{0}}-Y^{4} \frac{k \varepsilon \chi_{B}}{24 C_{k} C_{\mu} \bar{U} Y_{0}}+Y^{2}\left(-\frac{k \omega_{n f} \varepsilon}{2 C_{k}}+a \frac{k \varepsilon \chi_{B}}{2 C_{k} \bar{U} Y_{0}}\right)+B_{3} \\
+B_{4} Y \\
\theta_{n f 2}(Y)=-\frac{k Y^{2}\left(C_{\mu} \bar{U} \omega_{s}+C_{\mu} \bar{U} \omega_{n f} Y_{0}-D a Y_{0} \chi_{B}\right)}{2 C_{\mu} \bar{U}\left(k+C_{k} Y_{0}\right)} \\
+B_{5} \frac{C_{k} Y_{0}^{2}}{B i\left(k+C_{k} Y_{0}\right)} \cosh (\alpha Y)+B_{6} \\
+c \frac{k\left(-B i+Y_{0} Z^{2}\right) \chi_{B}}{\bar{U} Z^{2}\left(-k B i-B i C_{k} Y_{0}+C_{k} Y_{0}^{2} Z^{2}\right)} \cosh (Z Y)
\end{gathered}
$$




$$
\begin{aligned}
\theta_{s}(Y)=-\frac{k Y^{2}\left(C_{\mu} \bar{U} \omega_{s}+C_{\mu} \bar{U} \omega_{n f} Y_{0}-\operatorname{Da} Y_{0} \chi_{B}\right)}{2 C_{\mu} \bar{U}\left(k+C_{k} Y_{0}\right)} \\
+B_{7} \frac{C_{k} Y_{0}^{2}}{B i\left(k+C_{k} Y_{0}\right)} \cosh (\alpha Y)+B_{8} \\
+c \frac{k B i \chi_{B}}{\bar{U} Z^{2}\left(k B i+B i C_{k} Y_{0}-C_{k} Y_{0}^{2} Z^{2}\right)} \cosh (Z Y)
\end{aligned}
$$

Where $\quad \chi_{A}=\left(1+Q_{1}\left(1-Y_{0}\right)+\omega_{n f} Y_{0}+\omega_{S} Y_{p}\right), \chi_{B}=\left(1+\left.\gamma_{p}\right|_{\text {Model } B}+Q_{1}\left(1-Y_{0}\right)+\right.$ $\left.\omega_{n f} Y_{0}+\omega_{s} Y_{p}\right), \alpha=\frac{\sqrt{B i} \sqrt{k+C_{k} Y_{0}}}{\sqrt{C_{k} Y_{0}}}$ and constants $a, b$ and $c$ are provided by Eq. (24). The resultant coefficients $A_{1}$ to $A_{8}$ and $B_{1}$ to $B_{8}$ are long and complicated algebraic expressions, which in the interest of maintaining clarity and conciseness are not provided here. Lastly, the general solution of the dispersion equations is given by the following relations,

$$
\begin{gathered}
\Phi_{1}(Y)=M_{1}+M_{2} Y-\operatorname{Sr}_{1} \theta_{n f 1}(Y), \\
\Phi_{2}(Y)=M_{3}-S r_{2} \theta_{n f 2}(Y) .
\end{gathered}
$$

The coefficients $M_{1}$ to $M_{3}$ are cumbersome and have not been provided here for the sake of brevity.

\section{Results and discussion}

\subsection{Validation}

To validate the model, all parameters were set such that the problem resembled those already investigated in the literature. In particular, the thickness of the walls was made negligible $\left(Y_{0} \approx 1\right)$, the catalytic layer and hence mass transfer were discarded, and the concentration of nanoparticles was reduced to zero. Hence, the predicted temperature fields could be compared with the work of Karimi et al. [51] and the calculated $\mathrm{Nu}$ became comparable to those presented in Ref. [33]. Figure 2 shows that graphs identical to those presented in the aforementioned references were successfully produced, thereby 
validating the analytical solutions developed in Section 2. Also, although not shown here, it was observed that in the limit of fully-filled microchannel and high Bi (thus LTE dominated), the observed trends in concentration profile follow those documented in the work of Matin and Pop [52].

\subsection{Temperature fields and Nusselt number}

Figures 3-6 depict the contribution of different parameters of the microreactor on the temperature distribution and $\mathrm{Nu}$. Figure 3 shows the effects of variations in the internal heat exchanges between the solid and nanofluid phases of the porous medium, i.e. $B i$, and nanofluid internal heat generation on the temperature field of the microreactor. A comparison between Figs. $3 \mathrm{a}$ and $3 \mathrm{~b}$ reveals that varying $\mathrm{Bi}$ in Models $\mathrm{A}$ and $\mathrm{B}$ has different effects on the temperature distribution. While the effects of varying $B i$ is observed only within the porous section in Model A, Bi variations under Model B have broader impact on the temperature distribution of the microchannel. Increasing $B i$ in Model A decreases the temperature difference between the nanofluid and solid phases of the porous media yet does not have any substantial influence upon the temperature distribution within the other parts of the microchannel. However, increasing $B i$ under Model B decreases the temperature difference between the nanofluid and solid phases of the porous section, and also reduces the value of dimensionless temperature within the other parts of the system. Figures $3 \mathrm{c}$ and $3 \mathrm{~d}$ show that by increasing the absolute value of the internal heat generation in the nanofluid phase of the microreactor, which is the manifestation of the exo/endothermicity of the homogenous reactions through the nanofluid, the temperature difference between the solid and nanofluid phases increases. For Model B, even without internal heat generation within nanofluid phases of the system, the temperature difference between the two phases of the porous medium is not negligible. Hence, as also shown previously [20], this is a strong evidence showing the 
necessity of taking an LTNE approach in the thermal analysis of microreactors which involve internal heat generations.

Figure 4 provides an insight into the effects of nanoparticles volumetric concentration (NVC) and thermal conductivity ratio parameters on the temperature distributions under Models A and B. Three different NVCs have been examined in addition to the base fluids, i.e. $\phi=0 \%$, in Figs. $4 \mathrm{a}$ and $4 \mathrm{~b}$. Both models show that increasing the NVC within the system decreases the temperature differences within the system and helps achieving a uniform temperature within the microchannel, which is a desired feature of microreactors. This is, as apparent, because increasing NVC increases the thermal conductivity of the system, and hence the temperature distributions within each two specific points of the systems tend to become closer to each other. Also, it is seen that adding nanoparticles to the available fluid within microchannel is more beneficial at lower concentrations on nanoparticles, as the dimensionless temperature of the microchannel decreases by adding more nanoparticles to the system. By keeping NVC at $\phi=4 \%$, Figs. $4 \mathrm{c}$ and $4 \mathrm{~d}$ show the effect of thermal conductivity ratio parameter $\mathrm{k}$ on the temperature distributions within the microchannel for Models A and B. Similar to the effect of NVC on the temperature distributions, the thermal conductivity ratio has a monotonic effect upon the temperature distribution across the wall and those of the open section and, the nanofluid and solid phases of the porous section of the microchannel. This means that, under both models, increasing the thermal conductivity ratio results in reducing the dimensionless temperature of the wall, increasing the temperature difference within the microchannel, and boosting the fluid and solid phases' temperatures within the porous section of the microchannel.

Figure 5 shows the effects of internal heat generation within solid walls and solid phase of the porous medium on the temperature distributions of the microreactor, using 
both Models A and B. Figures. 5a and 5b, indicate that increasing the internal heat generation within the solid walls of the microreactor, increases the dimensionless temperature across the walls of microreactor and the adjacent nanofluid. However, for both Models A and B, this increment may not go beyond open section of the microreactor and is not observed in the porous sections. Similar to those of exo/endothermicity of the nanofluid on the temperature distributions, the effects of internal heat generation within the solid phase of the porous medium are seen on all parts of the system. This again endorses the suitability of the LTNE model for thermal analyses of thermochemical microreactors.

Figure 6 shows the effects of $\mathrm{Bi}$ and NVC on $\mathrm{Nu}$ for Models A and B, while varying the porous thickness value and keeping the other parameters fixed. This figure shows that depending on the value of $B i$, changes of $Y_{p}$ under Model A may result in one or two extremum(s) for $\mathrm{Nu}$. However, under Model B, varying the value of $Y_{p}$ from 0 to 0.8 only gives one maximum value for $N u$. This can be seen by comparing Figs. $6 \mathrm{a}$ and $6 \mathrm{~b}$. Moreover, changing the value of $\mathrm{Bi}$ under Model A has greater impact on $\mathrm{Nu}$ compared that under Model B. However, for both models and with the used parametric values in this figure, $Y_{p} \approx 0.6$ represents the optimum porous thickness to achieve the maximum value of $\mathrm{Nu}$. Figures $6 \mathrm{c}$ and $6 \mathrm{~d}$ illustrate the effects of NVC on $\mathrm{Nu}-Y_{p}$ plots for both models. A similar behaviour regarding the number of extrema, but in a less obvious way, can be seen in this set of figures as well. Depending on the value of NVC on Model A, one or two extremum(s) can be observed. Also, as expected, increasing NVC results in obtaining higher $\mathrm{Nu}$ for both models.

\subsection{Concentration fields}

Figures 7-9 illustrate the effects of various characteristics of microreactor upon the chemical species concentration within the porous and open sections of the microchannel. 
Figure 7 shows the effects of $D a$ and the diffusion coefficient ratio $\left(D_{21}\right)$ on the concentration of the chemical species within the microreactor. The effects of $D a$, which impact the concentration plots via Soret number, is clearly seen for both Models A and B. Increasing $D a$ value results in slower flow field, which ultimately increases the temperature and gives more time to the species to disperse within the domain. Hence, for both cases increasing $D a$ increases the concentration rate. Although increasing $D a$ results in enhancement of the dimensionless concentration for both interface models, its impact on the concentration of chemical species is more pronounced under Model A. Increasing Da from $10^{-5}$ to $10^{-3}$ in Model A, increases the dimensionless concentration at the centre of the microreactor from 0.65 to 0.9 , while for Model B this increment is from 0.65 to 0.8 . This behaviour remains unchanged in Figs. $7 \mathrm{c}$ and $7 \mathrm{~d}$ which illustrate the impacts of $D_{21}$ on the concentration of the chemical species. As $D_{21}$ increases, the dimensionless concentration for both interface models increases, with more noticeable impact when Model A is in place.

Figure 8 shows the effects of NVC on the dimensionless concentration when $S r_{1}$ and $S r_{2}$ are kept at 0.2 for both interface models. As expected, increasing NVC decreases the temperature distribution. Hence the non-dimensional concentration, which is coupled with the temperature distribution via Soret numbers, drops through increasing NVC. This is almost the same phenomenon observed in Fig. 9a. Since the effect of increasing NVC from $0 \%$ to $2 \%$ on the temperature distribution is more than the effect of increasing NVC from $4 \%$ to $6 \%$, this difference has shown itself with the dimensionless concentration values in Fig. 8. As it is clearly seen, the differences between the lines decreases by increasing the NVC. Compared with Figs. 7 and 8, in both Figs. 9a and $9 \mathrm{~b}$ the dimensionless concentration has been substantially increased because of the negative value for $\mathrm{Sr}_{2}$. This can be explained by noting that in Eq. (35) the second derivative of 
dimensionless temperature is also negative (see Figs. 3-5) and thus the thermal diffusion term effectively acts as a source term of the chemical species in this equation.

\subsection{Total entropy generation and PEC}

Figure 10 shows the effects of different parameters of the microchannel on the total entropy generation rate. This figure indicates that by modifying the value of a specific parameter it could be possible to minimize the total entropy generation. Further, for a given set of dimensionless parameters, the two interface models may predict different optimal points for minimisation of the total entropy. Through a close examination of the sub-figures, a few conclusions are drawn. Figure 10a shows that increasing $Y_{0}$, i.e. decreasing the wall's thickness, decreases the predicted total entropy generation rate. This means that by neglecting the wall effects on the temperature fields and consequently on the thermal irreversibility, the total entropy generation will be under-predicted. This is an important finding clearly demonstrating significance of inclusion of the solid walls of microsystems in their entropic analyses. Figures $10 \mathrm{c}$ to $10 \mathrm{e}$ show that it would be possible to find an optimum value regarding any heat consuming/generating part of the system to minimize the entropy generation rate. Yet, such optimal value depends upon the choice of the interface model. For example, it can be observed from Fig. 10c that when Model B is considered, by assuming $\omega_{n f}=-2$ and fixing other parameters, the total entropy generation rate has been minimized. Increasing the porous thickness or NVC monotonically increases the total entropy generation rate. Further, due to the reduction of friction irreversibility, increasing Darcy number decreases the total entropy generation within the microreactor.

Figures 11 and 12 show the $P E C$ of the microreactor versus porous thickness while varying the values of other parameters. Overall, unlike $N u$ versus $Y_{p}$ plots in which maximum $N u$ occurs at $Y_{p} \approx 0.6$, here the maximum performance occurs at different 
thickness of the porous insert depending on the values of other parameters. With used parametric values in this investigation, the optimum thickness can be as low as 0 to as high as 0.4 . However, the optimum porous thickness has not exceeded $Y_{p}=0.5$. By comparing the effects of wall's thickness and thermal conductivity ratio, it is inferred that the maximum PEC for both interface models does not change significantly by changing the wall's thickness. Yet, it changes drastically by changing the thermal conductivity ratio. A similar behaviour can be observed in Fig. 12. Although NVC appears to have marginal effects on $P E C$, changing $D a$ leaves influential impact on $P E C$.

\section{Conclusions}

An analytical investigation on the heat and mass transfer of a microreactor accommodating a first order, catalytic chemical reaction has been performed. A porous insert was placed along the centreline of the reactor, while the catalyst layer was located on the internal surface of walls. The finite thickness of the walls was considered and an LTNE approach was taken to analyse heat transfer in the porous section of microreactor. Further, the thermal diffusion of mass through Soret effect was considered and the dispersion of chemical species was analysed in the limit of small mass transfer Peclet number. Nanoparticles were used to augment heat transfer within the microreactor and two different models to describe fluid-porous interface conditions were applied. It was observed that, with the considered parametric values, the optimum thickness of the porous insert to achieve the maximum $\mathrm{Nu}$ is $Y_{p} \approx 0.6$. However, under each interface model PEC could be maximized by a different value of the thickness of the porous insert. The optimum value of this parameter to achieve the maximum PEC differs from 0 to 0.5 . It was also observed that, while the total entropy generation increases monotonically with thickening the porous insert, the total entropy generation can be optimized by choosing an optimum value for the endothermicity in each part of the system. This 
investigation shed some light on the energetic and entropic features of thick-walled microreactors, partially filled by porous inserts that have not been investigated previously. The provided analytical expressions and physical discussions are useful for the design and optimization of new microreactors. They can also serve as benchmark data for validation of the future theoretical and numerical investigations.

\section{References}

[1] Bejan A. Entropy Generation Through Heat and Fluid Flow. New York: Wiley; 1982.

[2] Bejan A. Advanced Engineering Thermodynamics. John Wiley \& Sons, Inc.; 2006.

[3] Naterer GF, Camberos JA. Entropy-Based Design and Analysis of Fluids Engineering Systems. CRC Press; 2008.

[4] Torabi M, Zhang K, Karimi N, Peterson GP. Entropy generation in thermal systems with solid structures - A concise review. Int J Heat Mass Transf 2016;97:917-31. doi:10.1016/j.ijheatmasstransfer.2016.03.007.

[5] Mahian O, Kianifar A, Kleinstreuer C, Al-Nimr MA, Pop I, Sahin AZ, et al. A review of entropy generation in nanofluid flow. Int J Heat Mass Transf 2013;65:514-32. doi:10.1016/j.ijheatmasstransfer.2013.06.010.

[6] Torabi M, Karimi N, Peterson GP, Yee S. Challenges and progress on the modeling of entropy generation in porous media: A review. Int J Heat Mass Transf 2017;114:31-46. doi:http://dx.doi.org/10.1016.

[7] Mies MJM, Rebrov E V., Deutz L, Kleijiv CR, De Croon MHJM, Schouten JC. Experimental validation of the performance of a microreactor for the highthroughput screening of catalytic coatings. Ind Eng Chem Res 2007;46:3922-31. doi:10.1021/ie061081w.

[8] Suryawanshi PL, Gumfekar SP, Bhanvase BA, Sonawane SH, Pimplapure MS. A review on microreactors: Reactor fabrication, design, and cutting-edge 
applications. Chem Eng Sci 2018;189:431-48. doi:10.1016/j.ces.2018.03.026.

[9] Turkakar G, Okutucu-Ozyurt T, Kandlikar SG. Entropy generation analysis of a microchannel-condenser for use in a vapor compression refrigeration cycle Göker. Int J Refrig 2016;70:71-83. doi:10.1016/j.ijrefrig.2016.06.028.

[10] Semenic T, You SM. Two-Phase Heat Sinks with Microporous Coating. Heat Transf Eng 2013;34:246-57. doi:10.1080/01457632.2013.703572.

[11] Hunt G, Karimi N, Torabi M. Two-dimensional analytical investigation of coupled heat and mass transfer and entropy generation in a porous, catalytic microreactor. Int J Heat Mass Transf 2018;119:372-91. doi:10.1016/j.ijheatmasstransfer.2017.11.118.

[12] Xia G, Ma D, Zhai Y, Li Y, Liu R, Du M. Experimental and numerical study of fluid flow and heat transfer characteristics in microchannel heat sink with complex structure. Energy Convers Manag 2015;105:848-57. doi:10.1016/j.enconman.2015.08.042.

[13] Dixit T, Ghosh I. Review of micro- and mini-channel heat sinks and heat exchangers for single phase fluids. Renew Sustain Energy Rev 2015;41:1298-311. doi:10.1016/j.rser.2014.09.024.

[14] Sun Y, Zhang L, Xu H, Zhong X. Subcooled flow boiling heat transfer from microporous surfaces in a small channel. Int J Therm Sci 2011;50:881-9. doi:10.1016/j.ijthermalsci.2011.01.019.

[15] Mondal M, Misra RP, De S. Combined electroosmotic and pressure driven flow in a microchannel at high zeta potential and overlapping electrical double layer. Int J Therm Sci 2014;86:48-59. doi:10.1016/j.ijthermalsci.2014.06.029.

[16] Chen CH. Fully-developed thermal transport in combined electroosmotic and pressure driven flow of power-law fluids in microchannels. Int J Heat Mass Transf 
2012;55:2173-83. doi:10.1016/j.ijheatmasstransfer.2011.12.022.

[17] Chein R, Chen Y, Zhu H, Chung JN. Numerical Simulation of Flow Disturbance and Heat Transfer Effects on the Methanol-Steam Reforming in Miniature Annulus Type Reformers. Energy \& Fuels 2012;26:1202-13. doi:10.1021/ef201498t.

[18] Delsman ER, de Croon MHJM, Elzinga GD, Cobden PD, Kramer GJ, Schouten JC. The influence of differences between microchannels on microreactor performance. Chem Eng Technol 2005;28:367-75. doi:10.1002/ceat.200407126.

[19] Wang F, Huang J, Xu J. Continuous-flow synthesis of azo dyes in a microreactor system. Chem Eng Process - Process Intensif 2018;127:43-9. doi:10.1016/j.cep.2018.03.014.

[20] Hunt G, Torabi M, Govone L, Karimi N, Mehdizadeh A. Two-dimensional heat and mass transfer and thermodynamic analyses of porous microreactors with Soret and thermal radiation effects-An analytical approach. Chem Eng Process Process Intensif 2018;126. doi:10.1016/j.cep.2018.02.025.

[21] Yue J. Multiphase flow processing in microreactors combined with heterogeneous catalysis for efficient and sustainable chemical synthesis. Catal Today 2017;308:3-19. doi:10.1016/j.cattod.2017.09.041.

[22] Haber J, Kashid MN, Borhani N, Thome J, Krtschil U, Renken A, et al. Infrared imaging of temperature profiles in microreactors for fast and exothermic reactions. Chem Eng J 2013;214:97-105. doi:10.1016/j.cej.2012.10.021.

[23] Wang K, Lu YC, Xia Y, Shao HW, Luo GS. Kinetics research on fast exothermic reaction between cyclohexanecarboxylic acid and oleum in microreactor. Chem Eng J 2011;169:290-8. doi:10.1016/j.cej.2011.02.072.

[24] Kolb G. Review: Microstructured reactors for distributed and renewable production of fuels and electrical energy. Chem Eng Process Process Intensif 
2013;65:1-44. doi:10.1016/j.cep.2012.10.015.

[25] Pennemann H, Kolb G. Review: Microstructured reactors as efficient tool for the operation of selective oxidation reactions. Catal Today 2016;278:3-21. doi:10.1016/j.cattod.2016.04.032.

[26] Hooman K. Entropy generation for microscale forced convection: Effects of different thermal boundary conditions, velocity slip, temperature jump, viscous dissipation, and duct geometry. Int Commun Heat Mass Transf 2007;34:945-57. doi:10.1016/j.icheatmasstransfer.2007.05.019.

[27] Ibáñez G, López A, Pantoja J, Moreira J. Entropy generation analysis of a nanofluid flow in MHD porous microchannel with hydrodynamic slip and thermal radiation. Int J Heat Mass Transf 2016;100:89-97. doi:10.1016/j.ijheatmasstransfer.2016.04.089.

[28] Torabi M, Karimi N, Zhang K. Heat transfer and second law analyses of forced convection in a channel partially filled by porous media and featuring internal heat sources. Energy 2015;93:106-27. doi:10.1016/j.energy.2015.09.010.

[29] Torabi M, Zhang K. Temperature distribution, local and total entropy generation analyses in MHD porous channels with thick walls. Energy 2015;87:540-54. doi:10.1016/j.energy.2015.05.009.

[30] Hunt G, Karimi N, Torabi M. Analytical investigation of heat transfer and classical entropy generation in microreactors - The influences of exothermicity and asymmetry. Appl Therm Eng 2017;119:403-24. doi:10.1016/j.applthermaleng.2017.03.057.

[31] Elliott A, Torabi M, Karimi N. Thermodynamics analyses of porous microchannels with asymmetric thick walls and exothermicity: An entropic model of microreactors. J Therm Sci Eng Appl 2017;9:041013. doi:10.1115/1.4036802. 
[32] Torabi M, Torabi M, Peterson GP. Entropy generation of double diffusive forced convection in porous channels with thick walls and Soret effect. Entropy 2017;19:171. doi:10.3390/e19040171.

[33] Mahmoudi Y, Karimi N, Mazaheri K. Analytical investigation of heat transfer enhancement in a channel partially filled with a porous material under local thermal non-equilibrium condition: Effects of different thermal boundary conditions at the porous-fluid interface. Int J Heat Mass Transf 2014;70:875-91. doi:10.1016/j.ijheatmasstransfer.2013.11.048.

[34] Wang K, Vafai K, Wang D. Analytical characterization of gaseous slip flow and heat transport through a parallel-plate microchannel with a centered porous substrate. Int J Numer Methods Heat Fluid Flow 2016;26:854-78. doi:10.1108/HFF-09-2015-0364.

[35] Siavashi M, Talesh Bahrami HR, Saffari H. Numerical investigation of flow characteristics, heat transfer and entropy generation of nanofluid flow inside an annular pipe partially or completely filled with porous media using two-phase mixture model. Energy 2015;93:2451-66. doi:10.1016/j.energy.2015.10.100.

[36] Siavashi M, Talesh Bahrami HR, Aminian E. Optimization of heat transfer enhancement and pumping power of a heat exchanger tube using nanofluid with gradient and multi-layered porous foams. Appl Therm Eng 2018;138:465-74. doi:10.1016/j.applthermaleng.2018.04.066.

[37] Jiang PX, Fan MH, Si GS, Ren ZP. Thermal-hydraulic performance of small scale micro-channel and porous-media heat-exchangers. Int J Heat Mass Transf 2001;44:1039-51. doi:10.1016/S0017-9310(00)00169-1.

[38] Munirathinam R, Huskens J, Verboom W. Supported catalysis in continuous-flow microreactors. Adv Synth Catal 2015;357:1093-123. 
doi:10.1002/adsc.201401081.

[39] Govone L, Torabi M, Hunt G, Karimi N. Non-equilibrium thermodynamic analysis of double diffusive, nanofluid forced convection in catalytic microreactors with radiation effects. Entropy 2017;19. doi:10.3390/e19120690.

[40] Siavashi M, Bordbar V, Rahnama P. Heat transfer and entropy generation study of non-Darcy double-diffusive natural convection in inclined porous enclosures with different source configurations. Appl Therm Eng 2017;110:1462-75. doi:10.1016/j.applthermaleng.2016.09.060.

[41] Siavashi M, Ghasemi K, Yousofvand R, Derakhshan S. Computational analysis of SWCNH nanofluid-based direct absorption solar collector with a metal sheet. Sol Energy 2018;170:252-62. doi:10.1016/j.solener.2018.05.020.

[42] Torabi M, Zhang K, Yang G, Wang J, Wu P. Heat transfer and entropy generation analyses in a channel partially filled with porous media using local thermal nonequilibrium model. Energy 2015;82:922-38. doi:10.1016/j.energy.2015.01.102.

[43] Dickson C, Torabi M, Karimi N. First and second law analyses of nanofluid forced convection in a partially-filled porous channel - The effects of local thermal nonequilibrium and internal heat sources. Appl Therm Eng 2016;103:459-80. doi:10.1016/j.applthermaleng.2016.04.095.

[44] Xu H, Zhao C, Vafai K. Analytical study of flow and heat transfer in an annular porous medium subject to asymmetrical heat fluxes. Heat Mass Transf 2017;53:114. doi:10.1007/s00231-017-2011-X.

[45] Govone L, Torabi M, Wang L, Karimi N. Effects of nanofluid and radiative heat transfer on the double-diffusive forced convection in microreactors. J Therm Anal Calorim 2018. doi:10.1007/s10973-018-7027-z.

[46] Mashaei PR, Shahryari M, Madani S. Numerical hydrothermal analysis of water- 
Al203 nanofluid forced convection in a narrow annulus filled by porous medium considering variable properties: Application to cylindrical heat pipes. J Therm Anal Calorim 2016;126:891-904. doi:10.1007/s10973-016-5550-3.

[47] Wang K, Tavakkoli F, Vafai K. Analysis of gaseous slip flow in a porous microannulus under local thermal non-equilibrium condition - An exact solution. Int J Heat Mass Transf 2015;89:1331-41.

doi:10.1016/j.ijheatmasstransfer.2015.06.001.

[48] Alazmi B, Vafai K. Constant wall heat flux boundary conditions in porous media under local thermal non-equilibrium conditions. Int J Heat Mass Transf 2002;45:3071-87.

[49] Yang K, Vafai K. Analysis of heat flux bifurcation inside porous media incorporating inertial and dispersion effects - An exact solution. Int J Heat Mass Transf 2011;54:5286-97. doi:10.1016/j.ijheatmasstransfer.2011.08.014.

[50] Torabi M, Torabi M, Ghiaasiaan SM, Peterson GP. The effect of $\mathrm{Al}_{2} \mathrm{O}_{3}$-water nanofluid on the heat transfer and entropy generation of laminar forced convection through isotropic porous media. Int J Heat Mass Transf 2017;111:804-16. doi:10.1016/j.ijheatmasstransfer.2017.04.041.

[51] Karimi N, Agbo D, Talat Khan A, Younger PL. On the effects of exothermicity and endothermicity upon the temperature fields in a partially-filled porous channel. Int J Therm Sci 2015;96:128-48. doi:10.1016/j.ijthermalsci.2015.05.002.

[52] Matin MH, Pop I. Forced convection heat and mass transfer flow of a nanofluid through a porous channel with a first order chemical reaction on the wall. Int Commun Heat Mass Transf 2013;46:134-41.

doi:10.1016/j.icheatmasstransfer.2013.05.001. 


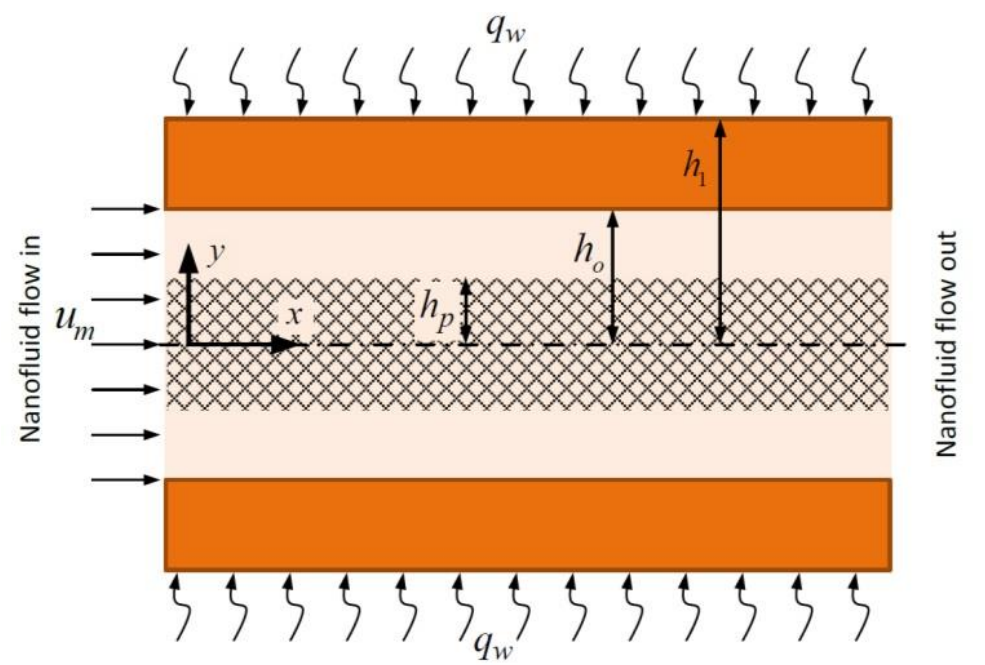

Fig. 1. Schematic configuration of the model microreactor. 

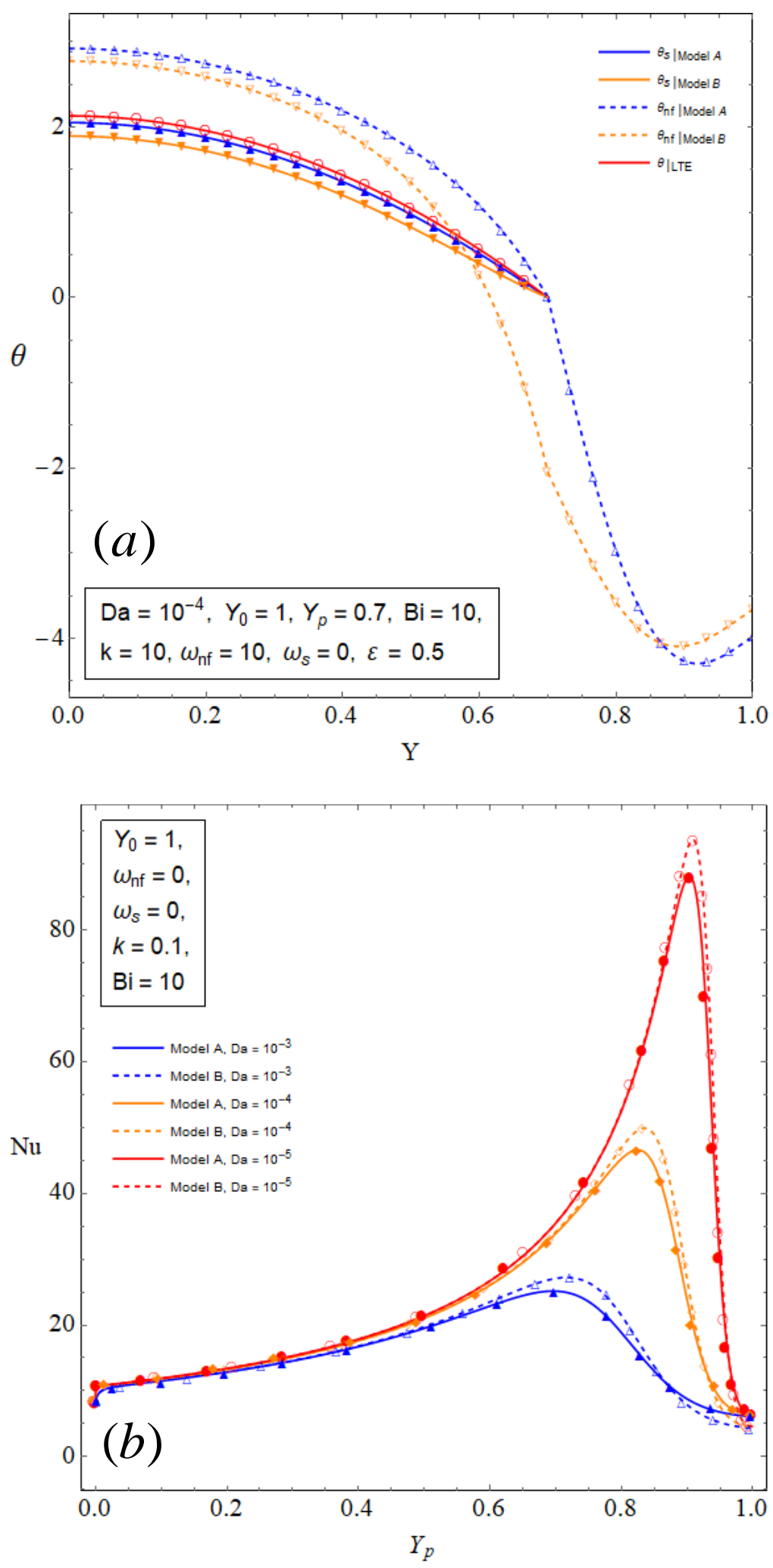

Fig. 2. Comparison of the present solution (symbols) with $(a)$ temperature fields of Ref.

[51] (lines) and (b) Nu of Ref. [33] (lines) for both interface models. 

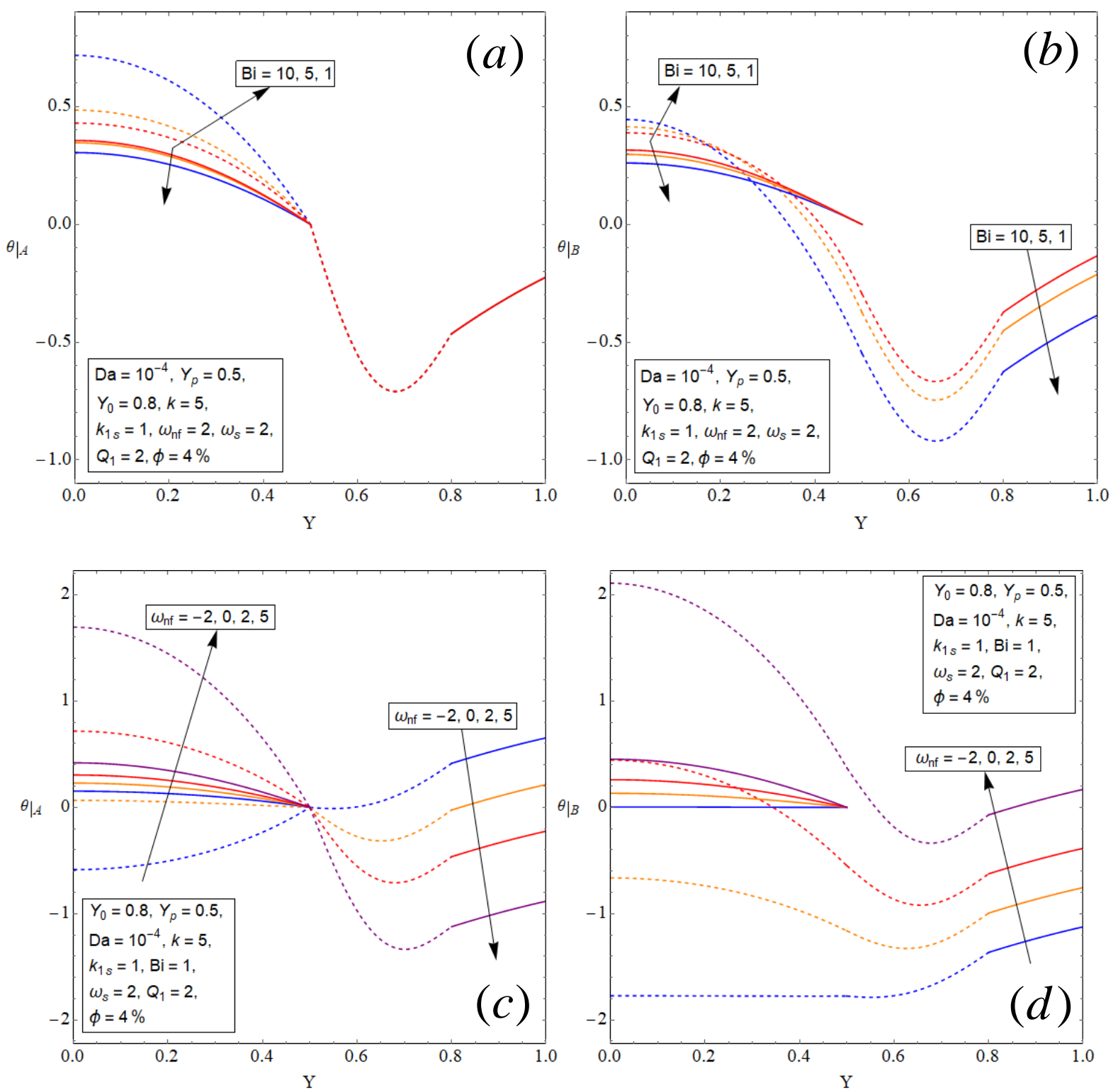

Fig. 3. Temperature variation for various values of $(a, b)$ Biot number and $(c, d)$ internal heat generation of the nanofluid phase for both Models A and B. 

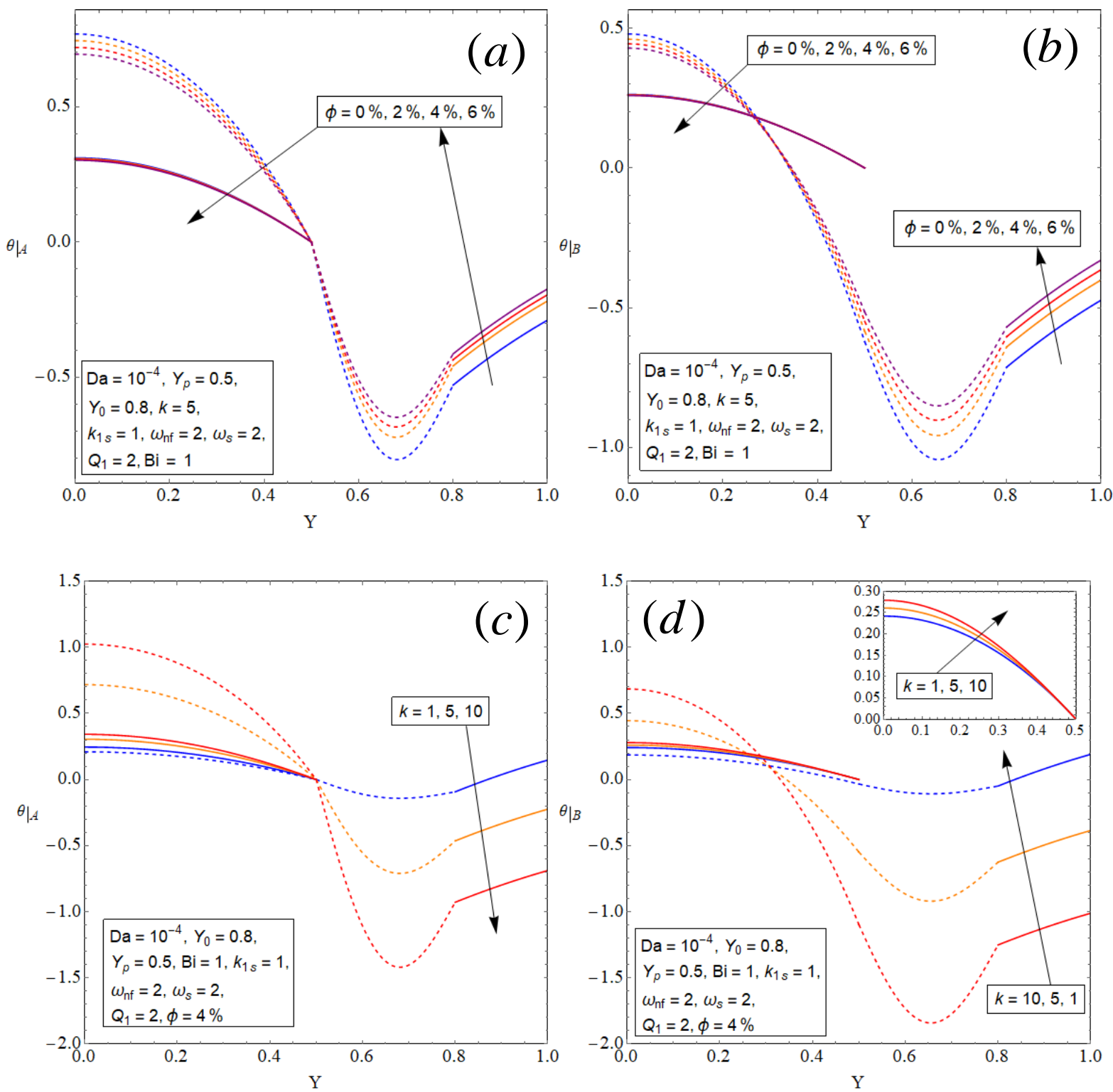

Fig. 4. Temperature variation for various values of (a,b) nanoparticles volumetric concentration and (c,d) thermal conductivity ratio for both Models A and B. 

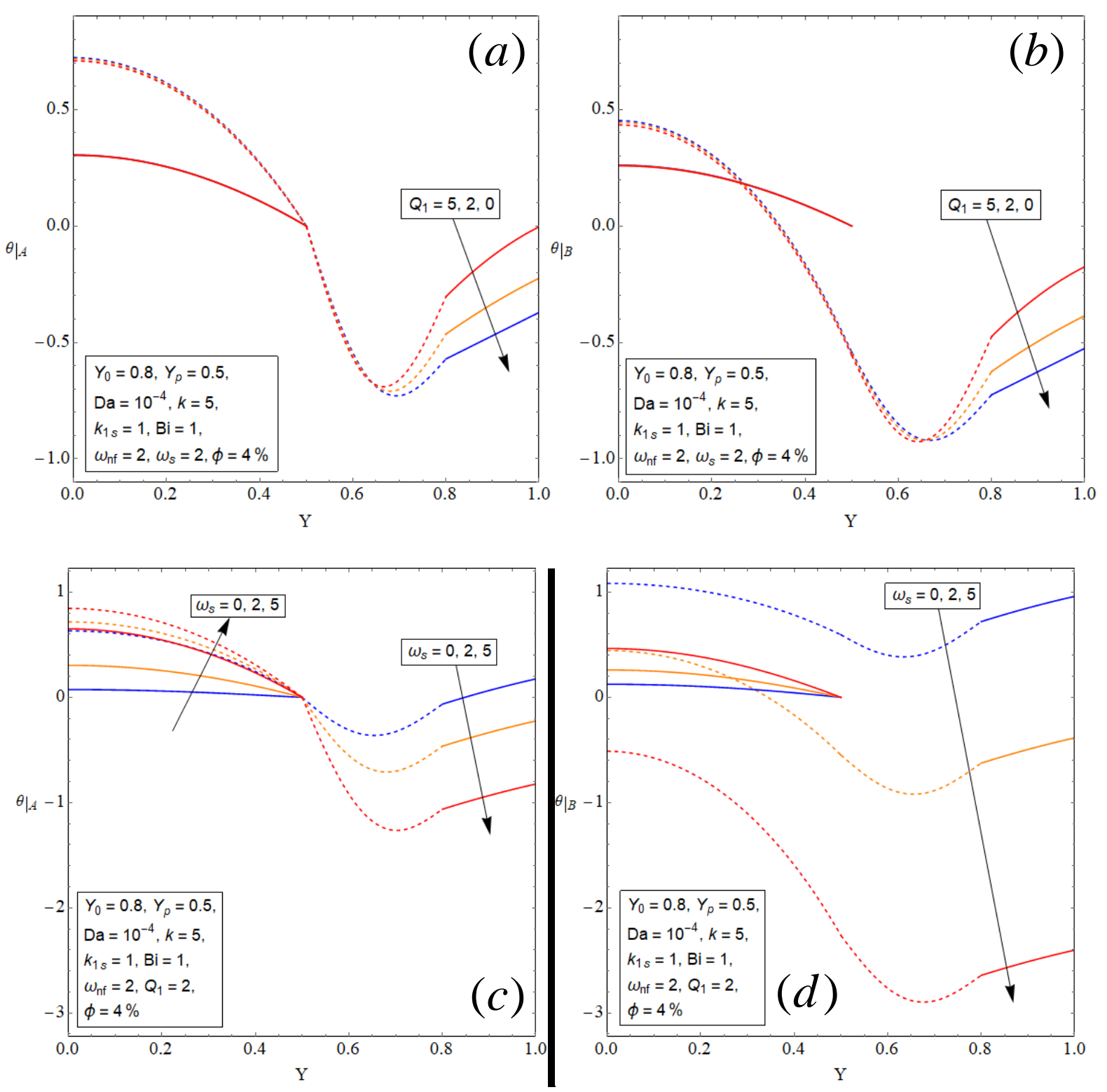

Fig. 5. Temperature variation for various values of internal heat

generation/consumption in $(\mathrm{a}, \mathrm{b})$ solid walls and $(\mathrm{c}, \mathrm{d})$ solid phases of the porous medium for both Models A and B. 

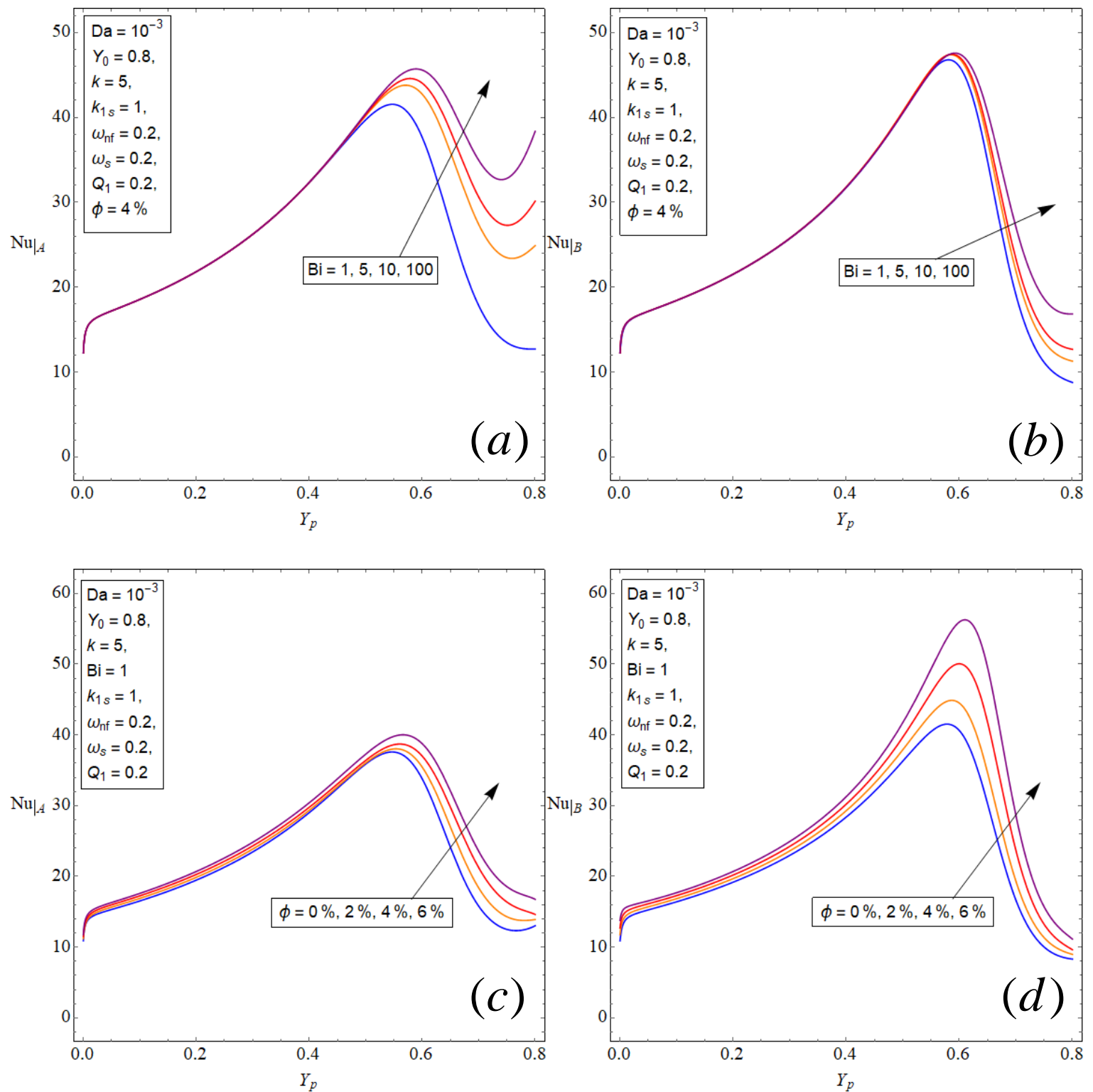

Fig. 6. Nusselt number versus porous thickness for various values of (a,b) Biot number and (c,d) nanoparticles volumetric concentration for both Models A and B. 

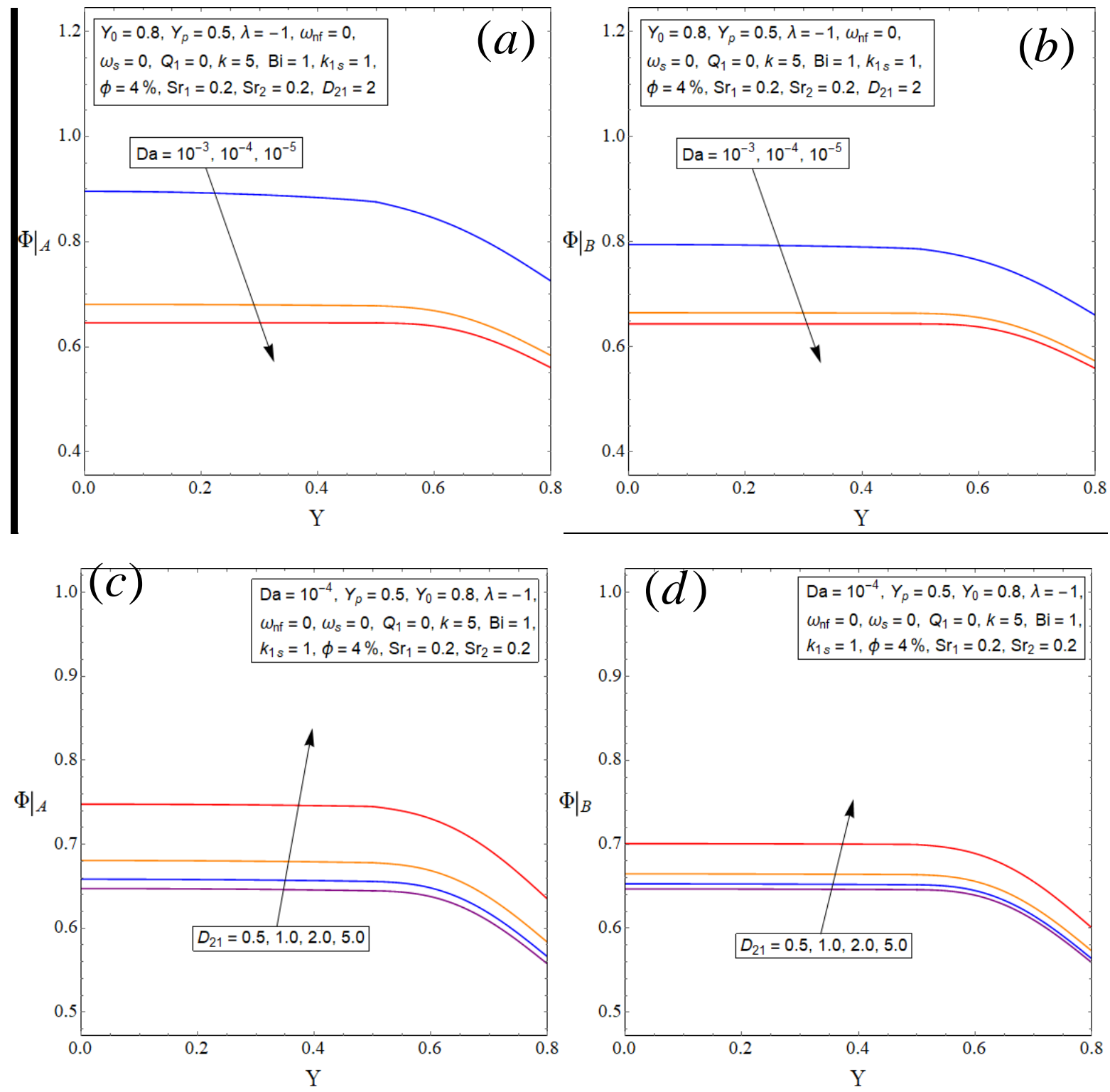

Fig. 7. Dimensionless concentration profiles for various values of $(a, b)$ Darcy number and (c,d) diffusion coefficient ratio for both Models A and B. 

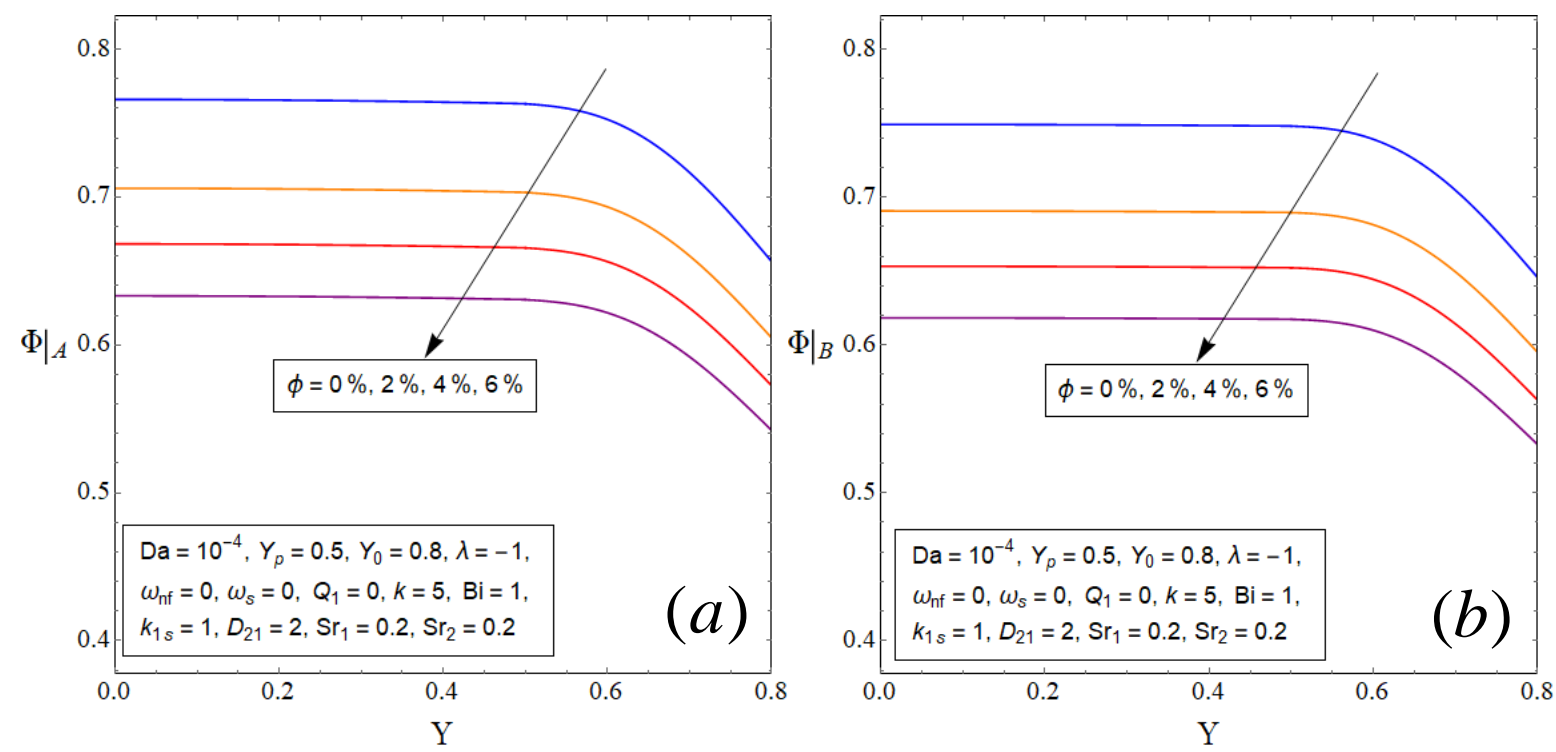

Fig. 8. Dimensionless concentration profiles for various values of nanoparticles volumetric concentration for both Models A and B. 

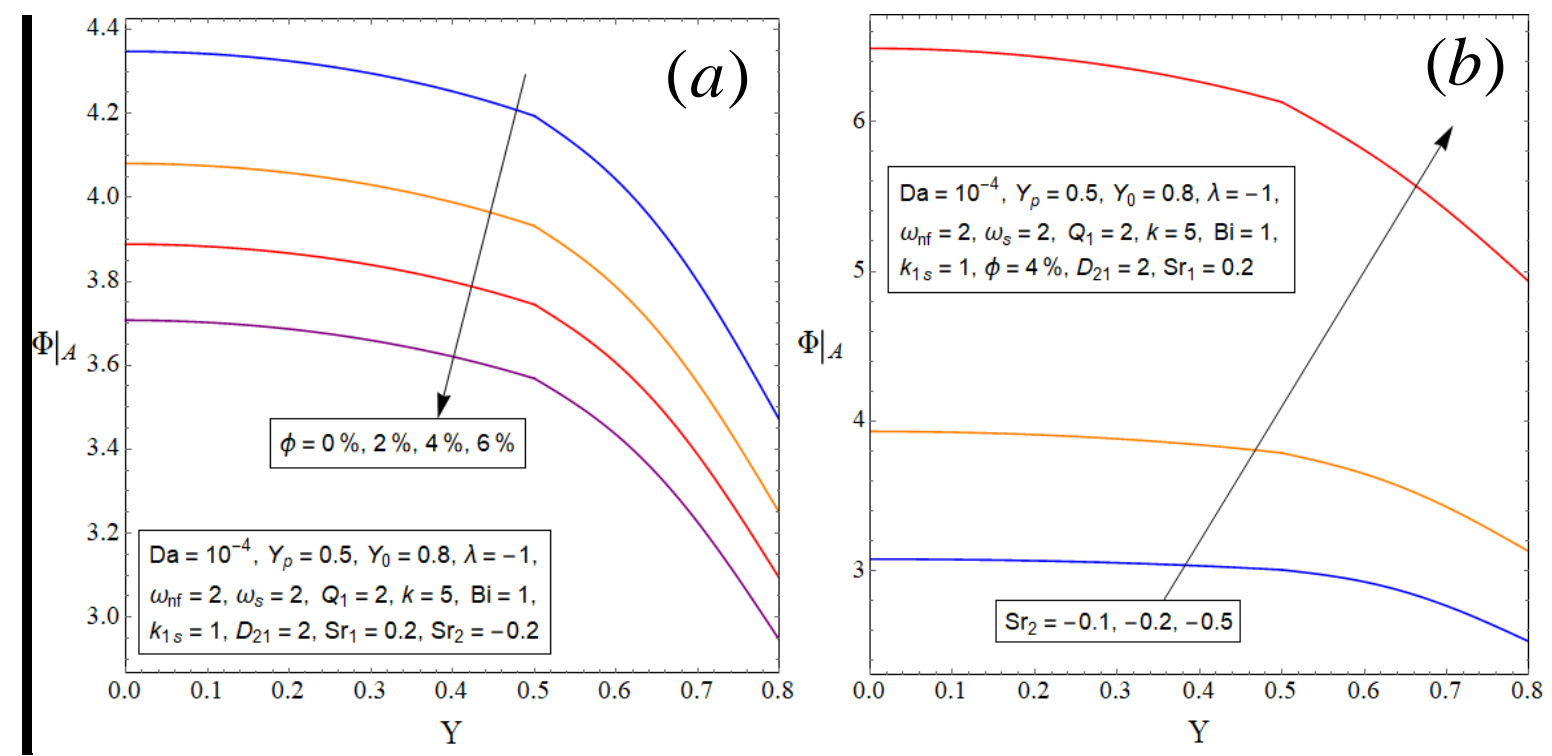

Fig. 9. Dimensionless concentration profile for various values of (a) nanoparticles volumetric concentration and (b) Soret number in porous section of the microreactor for both Models A and B. 

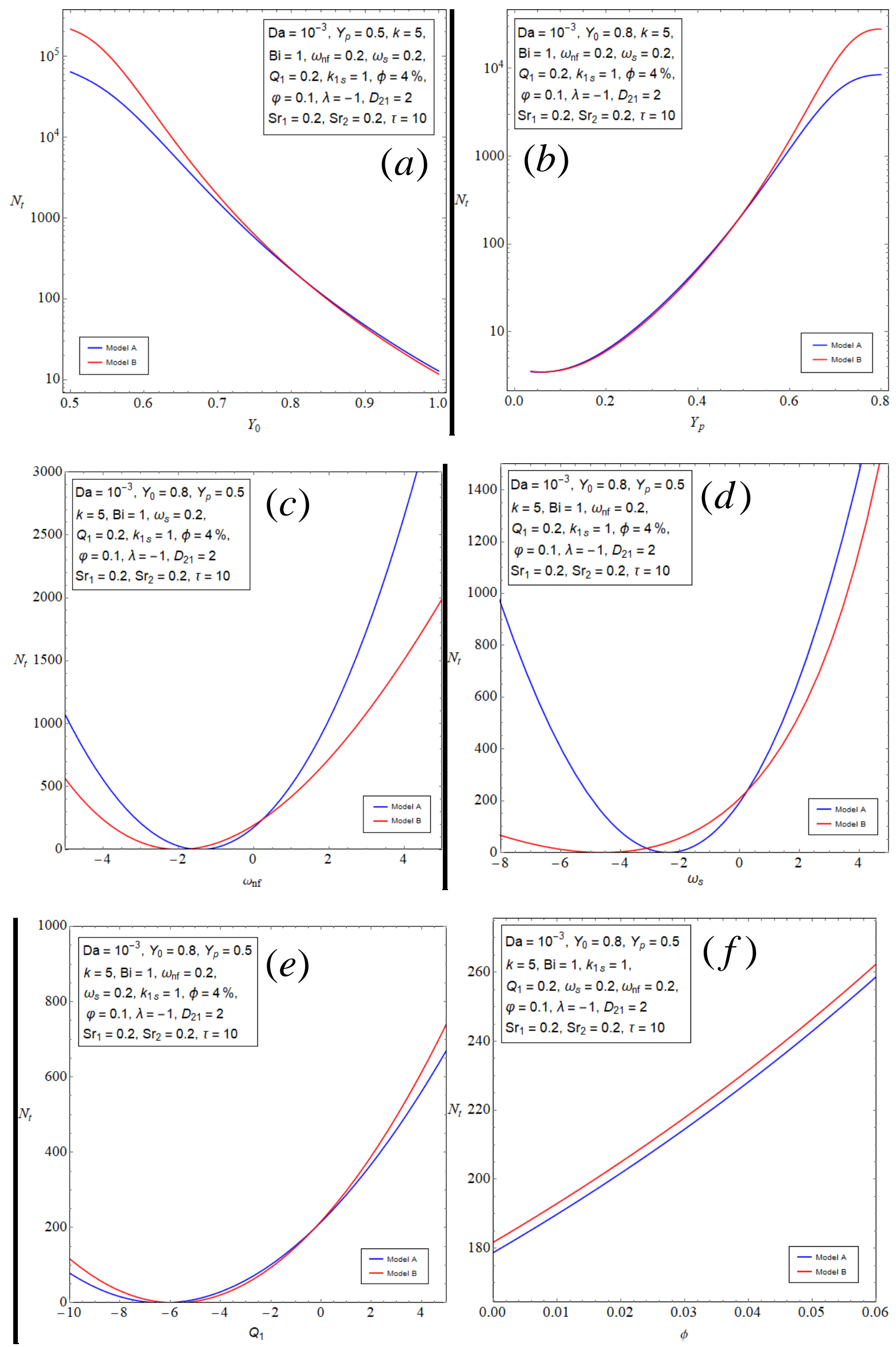


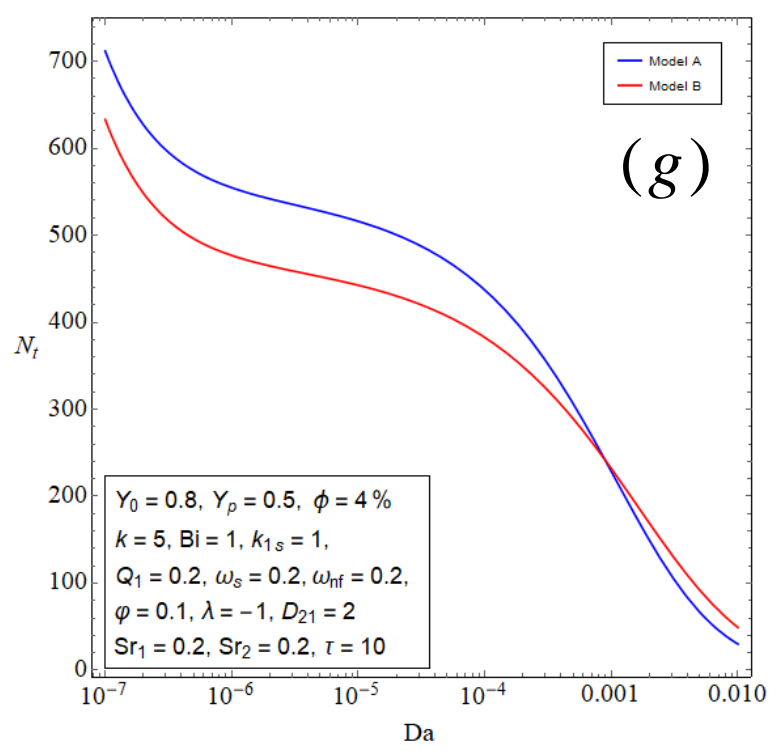

Fig. 10. Dimensionless total entropy generation rate versus $(a)$ wall's thickness, $(b)$ thickness of the porous insert, $(c)$ heat generation/consumption in the nanofluid phase, (d) heat generation/consumption in the solid phase of the porous section, $(e)$ heat generation/consumption in the wall, $(f)$ nanoparticles volumetric concentration and $(g)$ $D a$, for Models A and B. 

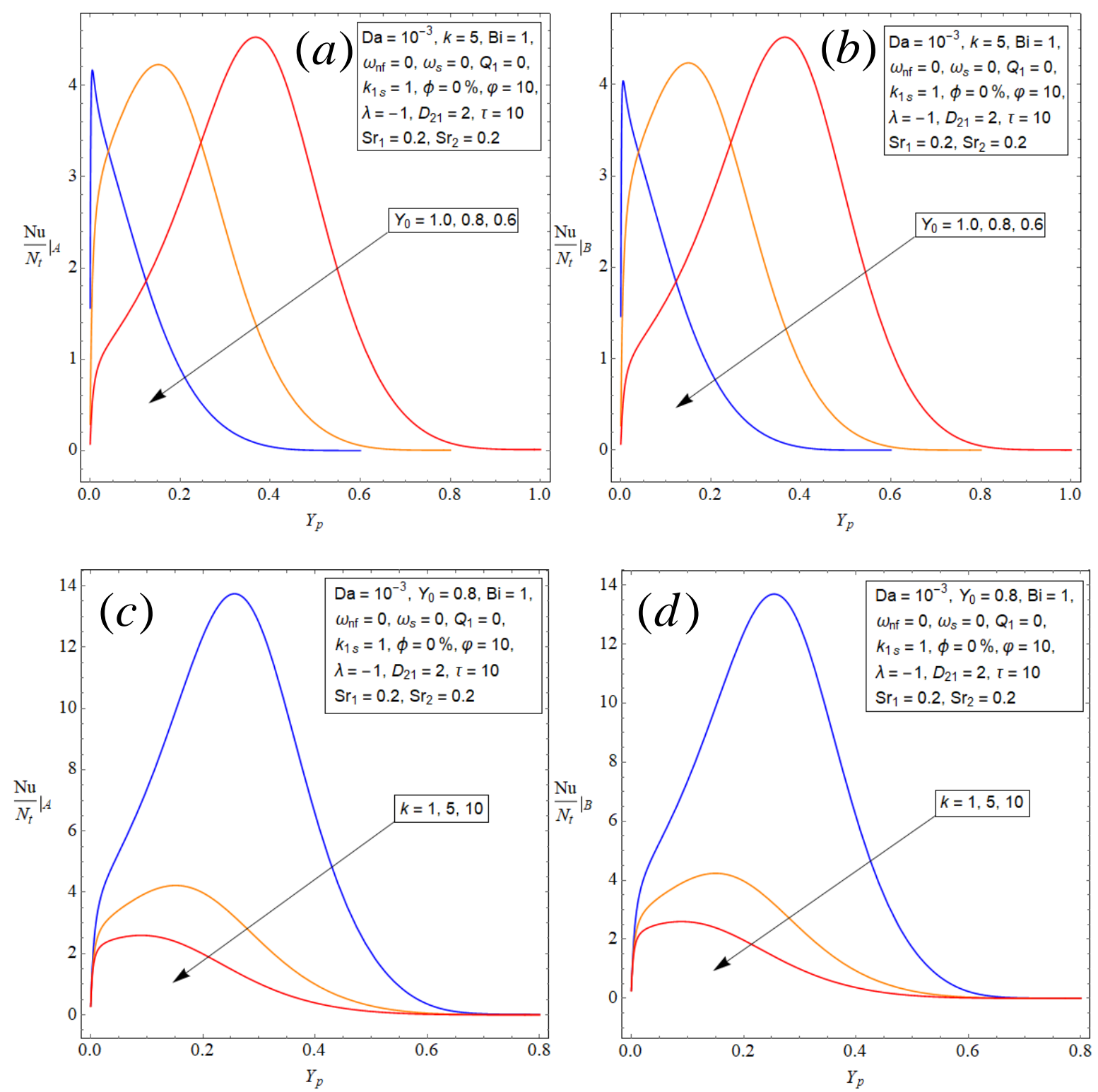

Fig. 11. Performance evaluation criterion versus thickness of the porous insert for various values of $(a, b)$ wall's thickness and $(c, d)$ thermal conductivity ratio for both Models A and B. 

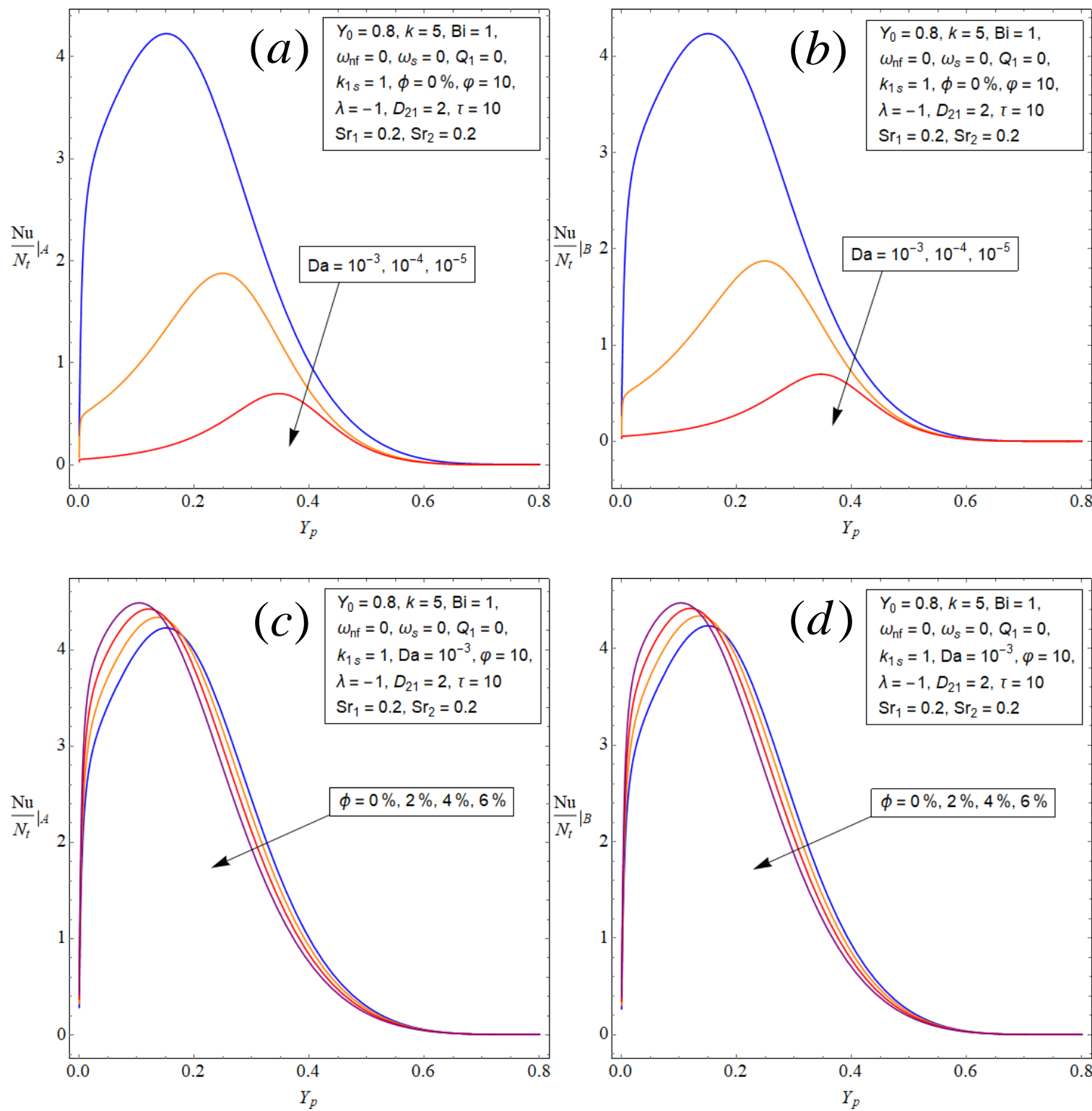

Fig. 12. Performance evaluation criterion versus porous thickness for various values of

(a,b) $D a$ and $(\mathrm{c}, \mathrm{d})$ nanoparticles volumetric concentration for both Models A and B. 